\title{
Localization of Floer homology of engulfed topological Hamiltonian loop
}

\author{
YONG-GEun OH*
}

In honor of Marshall Slemrod's 70-th birthday

Localization of Floer homology is first introduced by Floer [Fl2] in the context of Hamiltonian Floer homology. The author employed the notion in the Lagrangian context for the pair $\left(\phi_{H}^{1}(L), L\right)$ of compact Lagrangian submanifolds in tame symplectic manifolds $(M, \omega)$ in [Oh1, Oh2] for a compact Lagrangian submanifold $L$ and $C^{2}$-small Hamiltonian $H$. In this article, motivated by the study of topological Hamiltonian dynamics, we extend the localization process for any engulfed Hamiltonian path $\phi_{H}$ whose time-one map $\phi_{H}^{1}$ is sufficiently $C^{0}$-close to the identity (and also to the case of triangle product), and prove that the local Lagrangian spectral invariant defined on a Darboux-Weinstein neighborhood of $L$ is the same as the global one defined on the full cotangent bundle $T^{*} L$. Such a Hamiltonian path naturally occurs as an approximating sequence of engulfed topological Hamiltonian loop. We also apply this localization to the graph of $\phi_{H}^{t}$ in $(M \times M, \omega \oplus-\omega)$ and localize the Hamiltonian Floer complex of such a Hamiltonian $H$. We expect that this study will play an important role in the study of homotopy invariance of the spectral invariants of topological Hamiltonian.

KEYWORdS AND PHRASES: Local Floer homology, engulfed topological Hamiltonian loop, $J_{0}$-convex domain, maximum principle, thick-thin dichotomy, handle sliding lemma.

1 Introduction and the main results

1.1 Topological Hamiltonian loops

\subsection{Thick-thin dichotomy for $C^{0}$-small Lagrangian isotopy}

\subsection{Comparison of two Cauchy-Riemann equations}

*This work is supported by the Institute for Basic Sciences, and partially supported by US NSF grant \# DMS 0904197. 
1.4 Statement of main results 409

2 Local Floer chain module of engulfed Hamiltonian path $\phi_{H}$

3 Thick-thin dichotomy of Floer trajectories

4 Handle sliding lemma for engulfed isotopy of $C^{0}$-approximate loops

5 Computation of local Floer homology $H F_{*}^{[i d]}((L, L), H ; U) \quad 421$

6 Localization on the cotangent bundle

6.1 Localization of Lagrangian spectral invariants $\rho^{\operatorname{lag}(H ; 1)}$

6.2 Localization of the basic phase function

6.3 Localization of triangle product

A Local Floer complex of engulfed Hamiltonian $C^{0}$-approximate loop

A.1 Hamiltonian Floer complex

A.2 Isolating local Hamiltonian Floer complex

A.3 Fix $\phi_{G}^{1}$ versus $\Delta \cap \operatorname{graph} \phi_{G}^{1}$

\section{Introduction and the main results}

Construction of the local version of the Floer homology was introduced by Floer [Fl2]. The present author applied this construction to the Lagrangian context and defined the local Floer homology, denoted by $H F(H, L ; U)$, which singles out the contribution from the Floer trajectories whose images are contained in a given Darboux neighborhood $U$ of $L$ in $M$. Such an isolation of the contribution is proven to be possible and the resulting Floer homology is isomorphic to the singular homology $H_{*}(L)$ (with $Z_{2^{-}}$ coefficients) in [Oh2], provided $H$ is $C^{2}$-small. This $C^{2}$-smallness is used, conspicuously in $[\mathrm{Oh} 2]$, so that first

$$
\phi_{H^{t}}(L) \subset V \subset \bar{V} \subset U
$$

holds for all $t \in[0,1]$, and then the 'thick-thin' dichotomy of the Floer trajectories exists. The necessity of such a dichotomy is highlighted for the 
Floer moduli space of the boundary map, but its necessity is less conspicuous for that of the chain map in [Oh2].

But this latter was further scrutinized and exploited by Chekanov in his study of displacement energy in [Che1, Che2]. It follows from his argument in [Che1] that the quasi-isomorphism property of thin part of Floer chain maps between the local Floer complex $H$ and the Morse complex of $f$ holds for the Hamiltonian $H$ satisfying $\|H\|<\frac{1}{2} A\left(M, L, J_{0}\right)$ provided the thickthin dichotomy exists for the chain map. Here $A\left(M, L, J_{0}\right)$ is the smallest area of the non-constant $J_{0}$-holomorphic spheres or discs attached to $L$. (Chekanov denotes $\sigma\left(M, L, J_{0}\right)$ instead of $A\left(M, L, J_{0}\right)$.) The required thickthin dichotomy was established via the thick-thin dichotomy of associated Floer moduli spaces into those with big areas and those with very small areas. It was proved in [Oh2] that this dichotomy exists when $H$ is $C^{2}$ small by proving that all the thick trajectories have symplectic area greater than, say $\frac{1}{2} A\left(M, L, J_{0}\right)$, by a variation of Gromov-Floer compactness as $\phi_{H}^{1}(L) \rightarrow L$ in $C^{1}$-topology (or $H \rightarrow 0$ in $C^{2}$-topology). (We would like to emphasize that this convergence argument is not the standard Gromov-Floer type compactness argument since the limiting configuration is degenerate. The precise study of this convergence belongs to the realm of the so called adiabatic limit in the sense of [FO, Oh1, Oh7]. In [Oh2], it was enough to establish a non-constant component in the 'limit' which can be proved by a simple convergence argument under an energy bound.)

However such a dichotomy via the area does not exist when the convergence $\phi_{H}^{1}(L) \rightarrow L$ is assumed in $C^{0}$-topology (or $H \rightarrow 0$ in $C^{1}$-topology). We recall that understanding the behavior of various analytically constructed symplectic invariants via the Floer homology under the $C^{0}$ change of Hamiltonian diffeomorphisms is an important subject of study in symplectic topology. We now motivate such a study in relation to topological Hamiltonian dynamics á la $[\mathrm{OM}]$, [Oh9].

\subsection{Topological Hamiltonian loops}

In $[\mathrm{OM}]$, Müller and the author introduced the notion of hamiltonian topology on the space

$$
\mathcal{P}^{\text {ham }}(\operatorname{Symp}(M, \omega), i d)
$$

of Hamiltonian flows $\lambda:[0,1] \rightarrow \operatorname{Symp}(M, \omega)$ with $\lambda(t)=\phi_{H}^{t}$ for some timedependent Hamiltonian $H$. We first recall the definition of this hamiltonian topology. 
Following the notations of $[\mathrm{OM}]$, we denote by $\phi_{H}$ the Hamiltonian path

$$
\phi_{H}: t \mapsto \phi_{H}^{t} ;[0,1] \rightarrow \operatorname{Ham}(M, \omega)
$$

and by $\operatorname{Dev}(\lambda)$ the associated normalized Hamiltonian

$$
\operatorname{Dev}(\lambda):=\underline{H}, \quad \lambda=\phi_{H}
$$

where $\underline{H}$ is defined by

$$
\underline{H}(t, x)=H(t, x)-\frac{1}{\operatorname{vol}_{\omega}(M)} \int_{M} H(t, x) \omega^{n} .
$$

Definition 1.1. Let $(M, \omega)$ be a closed symplectic manifold. Let $\lambda, \mu$ be smooth Hamiltonian paths. The hamiltonian topology of Hamiltonian paths is the metric topology induced by the metric

$$
d_{\text {ham }}(\lambda, \mu):=\bar{d}(\lambda, \mu)+\operatorname{leng}\left(\lambda^{-1} \mu\right) .
$$

Now we recall the notion of topological Hamiltonian flows and Hamiltonian homeomorphisms (abbreviated as hameomorphisms) which was introduced in $[\mathrm{OM}]$.

Definition $1.2\left(L^{(1, \infty)}\right.$ topological Hamiltonian flow). A continuous map $\lambda: \mathbb{R} \rightarrow$ Homeo $(M)$ is called a topological Hamiltonian flow if there exists a sequence of smooth Hamiltonians $H_{i}: \mathbb{R} \times M \rightarrow \mathbb{R}$ satisfying the following:

(1) $\phi_{H_{i}} \rightarrow \lambda$ locally uniformly on $\mathbb{R} \times M$.

(2) the sequence $H_{i}$ is Cauchy in the $L^{(1, \infty)}$-topology locally in time and so has a limit $H_{\infty}$ lying in $L^{(1, \infty)}$ on any compact interval $[a, b]$.

We call any such $\phi_{H_{i}}$ or $H_{i}$ an approximating sequence of $\lambda$. We call a continuous path $\lambda:[a, b] \rightarrow H o m e o(M)$ a topological Hamiltonian path if it satisfies the same conditions with $\mathbb{R}$ replaced by $[a, b]$, and the limit $L^{(1, \infty)}$ function $H_{\infty}$ called a $L^{(1, \infty)}$ topological Hamiltonian or just a topological Hamiltonian.

We call a topological Hamiltonian path $\lambda$ a loop if $\lambda(0)=\lambda(1)$. Any approximating sequence $\phi_{H_{i}}$ of a topological Hamiltonian loop $\lambda$ has the property $\phi_{H_{i}}^{1} \rightarrow i d$ in addition to the properties (1), (2) of Definition 1.2. The following question is an important question to study in relation to the simpleness question of the area-preserving homeomorphism group of 2 disc $D^{2}$. 
Definition 1.3 (Hameotopy). Let $\lambda_{0}, \lambda_{1} \in \mathcal{P}^{\text {ham }}(\operatorname{Sympeo}(M, \omega), i d)$. A hameotopy $\Lambda:[0,1]^{2} \rightarrow \operatorname{Sympeo}(M, \omega)$ between $\lambda_{0}$ and $\lambda_{1}$ based at the identity is the map such that

$$
\Lambda(0, t)=\lambda_{0}(t), \Lambda(1, t)=\lambda_{1}(t),
$$

and $\Lambda(0, s) \equiv i d$ for all $s \in[0,1]$, and arises as follows: there is a sequence of smooth maps $\Lambda_{j}:[0,1]^{2} \rightarrow \operatorname{Ham}(M, \omega)$ that satisfy

(1) $\Lambda_{j}(s, 0)=i d$,

(2) $\Lambda_{j} \rightarrow \Lambda$ in $C^{0}$-topology,

(3) Any 'horizontal' section $\Lambda_{j, s}:\{s\} \times[0,1] \rightarrow \operatorname{Ham}(M, \omega)$ converges in hamiltonian topology in the following sense: If we write

$$
\operatorname{Dev}\left(\Lambda_{j, s} \Lambda_{j, 0}^{-1}\right)=: H_{j}(s),
$$

then $H_{j}(s)$ converges in hamiltonian topology uniformly over $s \in[0,1]$. We call any such $\Lambda_{j}$ an approximating sequence of $\Lambda$.

When $\lambda_{0}(1)=\lambda_{1}(1)=\psi$, a hameotopy relative to the ends is one that satisfies $\Lambda(s, 0)=i d, \Lambda(s, 1)=\psi$ for all $s \in[0,1]$ in addition.

We say that $\lambda_{0}, \lambda_{1} \in \mathcal{P}^{\text {ham }}(\operatorname{Sympeo}(M, \omega), i d)$ are hameotopic (resp. relative to the ends), if there exists a hameotopy (resp. a hameotopy relative to the ends).

We emphasize that by the requirement (3),

$$
H_{j}(0) \equiv 0
$$

in this definition. We say that a topological Hamiltonian loop contractible, if it is hameotopic to the constant loop.

All the above definitions can be modified to handle the case of open manifolds, either noncompact or compact with boundary, by considering $H$ 's compactly supported in the interior as done in section $6[\mathrm{OM}]$. We recall the definitions of topological Hamiltonian paths and Hamiltonian homeomorphisms supported in an open subset $U \subset M$ from $[\mathrm{OM}]$.

We first define $\mathcal{P}^{\text {ham }}\left(\operatorname{Symp}_{U}(M, \omega), i d\right)$ to be the set of smooth Hamiltonian paths supported in $U$. The following definition is taken from Definition $6.2[\mathrm{OM}]$ to which we refer readers for more detailed discussions. First for any open subset $V \subset U$ with compact closure $\bar{V} \subset U$, we can define the completion of $\mathcal{P}^{\text {ham }}\left(\operatorname{Symp}_{\bar{V}}(M, \omega), i d\right)$ using the same metric above which we denote by

$$
\mathcal{P}^{\text {ham }}\left(\operatorname{Symp}_{K}(M, \omega), i d\right), \quad K=\bar{V} .
$$


Definition 1.4. Let $U \subset M$ be an open subset. Define $\mathcal{P}^{\text {ham }}\left(\operatorname{Sympeo}_{U}(M, \omega), i d\right)$ to be the union

$$
\mathcal{P}^{\text {ham }}\left(\operatorname{Sympeo}_{U}(M, \omega), i d\right):=\bigcup_{K \subset U} \mathcal{P}^{\text {ham }}\left(\operatorname{Sympeo}_{K}(M, \omega), i d\right)
$$

with the direct limit topology, where $K \subset U$ is a compact subset. We define $\operatorname{Hameo}_{c}(U, \omega)$ to be the image

$$
\operatorname{Hameo}_{c}(U, \omega):=\operatorname{ev}_{1}\left(\mathcal{P}^{\text {ham }}\left(\operatorname{Sympeo}_{U}(M, \omega), i d\right) .\right.
$$

We would like to emphasize that this set is not necessarily the same as the set of $\lambda \in \mathcal{P}^{\text {ham }}(\operatorname{Sympeo}(M, \omega), i d)$ with compact $\operatorname{supp} \lambda \subset U$. The same definition can be applied to general open manifolds or manifolds with boundary.

Question 1.5. Let $H$ be a contractible topological Hamiltonian loop and let $\rho(H ; 1)$ be the spectral invariant defined by

$$
\rho(H ; 1)=\lim _{i \rightarrow \infty} \rho\left(H_{i} ; 1\right)
$$

for a (and so any) approximating sequence $H_{i}$. Is $\rho(H ; 1)=0$ ?

We refer to [Oh6] for the definition of spectral invariant $\rho(H ; 1)$ of smooth Hamiltonians $H$. Besides its intrinsic interest in $C^{0}$ symplectic topology, it has been shown by the author in [Oh9] and [Oh12] that the affirmative answer to this question implies that $\operatorname{Hameo}\left(D^{2}, \partial D^{2}\right)$ is a proper normal subgroup of the group $\operatorname{Homeo}^{\Omega}\left(D^{2}, \partial D^{2}\right)$ of area-preserving homeomorphisms of $D^{2}$ supported in the interior. This in particular implies that the latter group is not simple. Whether the latter group is simple or not is a prominent open problem in dynamical systems. (See [Oh9] for some history and ideas of the problem.)

Then in [Oh13], the author reduced the question to the case where the loop is sufficiently $C^{0}$-small so that the graph of the whole flow $\phi_{H}^{t}$ is contained in a (uniform) Darboux neighborhood of the diagonal in the product $M \times M$ and the Lagrangian spectral invariants of the $C^{0}$-small topological Hamiltonian deformations of the diagonal can be studied. This led us to study whether the Floer complex can be localized in a Darboux-Weinstein neighborhood in the way that the associated localized Lagrangian spectral invariant can be compared with the global Lagrangian spectral invariant in full cotangent bundle $T^{*} \Delta$. 


\subsection{Thick-thin dichotomy for $C^{0}$-small Lagrangian isotopy}

Motivated by the discussion laid out in the previous subsection, we are led to analyze the behaviors of the Floer moduli space and of the Floer complex as the boundary Lagrangian submanifold $\phi_{H_{i}}^{1}(L)$ for a sequence of Hamiltonian diffeomorphisms $\phi_{H_{i}}^{1} \rightarrow i d$ in $C^{0}$-topology.

Unlike the case of $C^{2}$-small Hamiltonians, the dichotomy via the areas described in the beginning of the present paper does not exist for the case of $C^{1}$-small Hamiltonian $H$. The main purpose of the present paper is to generalize the construction of local Floer homology and its computation for the case where the $C^{2}$-smallness of $H$ (or $C^{1}$-smallness of $\phi_{H}$ ) is replaced by the weaker hypothesis, the $C^{0}$-smallness of the time-one map $\phi_{H}^{1}: t \mapsto \phi_{H}^{t}$ for any engulfed Hamiltonian path $\phi_{H}$. There are two major differences between the cases of $C^{1}$-topology and of the $C^{0}$ topology of Hamiltonian paths (or between the $C^{2}$-smallness of $H$ and $C^{1}$-smallness of $\phi_{H}$ ). The first fundamental issue is that thin trajectories might not have small area but could have large area for the $C^{0}$-close Lagrangian submanifolds unlike the $C^{1}$-close case of Lagrangian submanifolds. Because of this, instead of using the areas as in [Oh2], we will use the maximum principle to single out 'thin' trajectories which turns out to be the best way of obtaining such a dichotomy even for the $C^{2}$-small $H$ 's in hindsight. However the thick-thin dichotomy acquired via the maximum principle does not differentiate the action filtration any more. The second more technical issue is that the $C^{0}$-topology is a priori too weak to uniformly control the analytical behavior of pseudo-holomorphic curves with boundary lying on $\phi_{H}^{1}(L)$ in general partially because we cannot establish uniform area bounds even for the thin trajectories, while $C^{1}$-topology of Lagrangian boundary condition controls analysis of pseudo-holomorphic curves.

To describe the problem in a precise manner, we need some digression.

Let $L \subset(M, \omega)$ be a compact Lagrangian submanifold and let $V \subset \bar{V} \subset$ $U$ be a pair of Darboux neighborhoods of $L$. We denote $\omega=-d \Theta$ on $U$ where $\Theta$ is the Liouville one-form on $U$ regarded as an open neighborhood of the zero section of $T^{*} L$. Following [Oh7], [Sp], we introduce the following notion.

We measure the size of the Darboux neighborhood $V$ by the following constant

$$
d(V, \Theta):=\max _{x \in V}|p(x)|, \quad x=(q(x), p(x)) .
$$


This constant is bounded away from 0 and so there exists some $\eta>0$ depending only on $(V,-d \Theta)$ (and so only on $(M, \omega))$ such that if $d_{C^{0}}\left(\phi_{H}^{1}, i d\right)<\eta$, then $\phi_{H}^{1}(L) \subset V$.

Definition 1.6. We call an isotopy of Lagrangian submanifold $\left\{L_{t}\right\}_{0 \leq s \leq 1}$ of $L$ is called $V$-engulfed if there exists a Darboux neighborhood $V$ of $L$ such that $L_{s} \subset V$ for all $s$. When we do not specify $V$, we just call the isotopy engulfed for $L$.

We call a (topological) Hamiltonian path $\phi_{H}$ engulfed if its graph Graph $\phi_{H}^{t}$ is engulfed in a Darboux neighborhood of the diagonal $\Delta$ of $(M \times M, \omega \oplus-\omega)$.

Following Weinstein's notation, we denote by $\mathfrak{I s o}(L)$ the set of Hamiltonian deformations of $L$. Define

$$
\begin{aligned}
\mathcal{H}_{\delta}^{\text {engulf }}(L ; V) & =\left\{H \mid \phi_{H}^{t}(L) \subset V \forall t \in[0,1], \bar{d}\left(\phi_{H}^{1}, i d\right) \leq \delta\right\} \\
\mathfrak{I s o}_{\delta}^{\text {engulf }}(L ; V) & =\left\{L^{\prime} \in \mathfrak{I s o}(L) \mid L^{\prime}=\phi_{H}^{1}(L), H \in \mathcal{H}_{\delta}^{\text {engulf }}(L ; V)\right\} .
\end{aligned}
$$

One of the main goals of the present paper is to extend the notion of local Floer homology introduced in [Fl2, Oh2] for the $C^{2}$-small Hamiltonian $H$ to the case of $H$ such that

(1) its Hamiltonian paths $\phi_{H}$ are $V$-engulfed,

(2) its time-one map $\phi_{H}^{1}$ is $C^{0}$-small.

Such a sequence of smooth Hamiltonian paths naturally occurs as an approximating sequence of engulfed topological Hamiltonian loop (based at the identity).

We would like to remark that it is established in [Oh2] that if $\|H\|_{C^{2}}<C$ for sufficiently small $C>0$, then the following automatically hold:

(1) its Hamiltonian paths $\phi_{H}$ is $V$-engulfed,

(2) and the uniform area bounds of the associated connecting Floer trajectories on $V$, where we regard $V$ as a neighborhood of the zero section in the cotangent bundle so that we use the classical action functional to measure the actions.

(3) The path spaces $\mathcal{P}\left(\phi_{H}^{1}(L), L\right)$ or $\mathcal{P}(L, L)$ carry a distinguished connected component on which the actions of any Hamiltonian chord become uniformly small.

In [Oh2], we mainly used the area of Floer trajectories to obtain the thickthin decomposition of the Floer boundary operator $\partial=\partial_{0}+\partial^{\prime}$, which is equivalent to the corresponding decomposition in terms of filtration changes 
under the boundary map (or the Floer chain map) for a $C^{2}$-small Hamiltonian $H$.

However for the Hamiltonian $H$ of our interest in the present paper, both properties (2) and (3) fail to uniformly hold even when we let $d_{C^{0}}\left(\phi_{H}^{1}, i d\right) \rightarrow$ 0 . Therefore there do neither exist a uniform gap in the filtration nor uniform control of the filtration of the Floer complex (or of the action bounds of the associated Hamiltonian chords). This is a new phenomenon for the localization in the current topological Hamiltonian context. Because of this lack of control of the filtration, we will instead use the more geometric version of thick-thin dichotomy mainly using the $C^{0}$ property of $\phi_{H}^{1}$ by exploiting the maximum principle.

For this purpose, we fix a time-independent almost complex structure $J_{0}$ that satisfies $J_{0} \equiv J_{g}$ on $V$ where $J_{g}$ is the canonical (Sasakian) almost complex structure on $V$ as a subset $T^{*} L$ which is induced by a Riemannian metric $g$ on $L$, and suitably interpolated to one defined on the complement of $U$. (We refer to [Fl3], [Oh2] for the precise description of $J_{g}$ and $J_{0}$ respectively.) We may assume $V$ has $J_{0}$-convex boundary. We denote by

$$
\mathcal{J}_{\omega}\left(V, J_{g}\right)
$$

the set of such almost complex structures.

\subsection{Comparison of two Cauchy-Riemann equations}

For each given pair $(J, H)$, we consider the perturbed Cauchy-Riemann equation

$$
\left\{\begin{array}{l}
\frac{\partial u}{\partial \tau}+J\left(\frac{\partial u}{\partial t}-X_{H}(u)\right)=0 \\
u(\tau, 0) u\left(\tau, L_{1}\right) \in L
\end{array}\right.
$$

which defines the Floer complex $C F_{*}(L, L ; H)$ generated (over a suitable Novikov ring) by the set $\mathcal{C} h o r d(H ; L, L)$ defined by

$$
\mathcal{C h o r d}(H ; L, L)):=\left\{z:[0,1] \rightarrow M \mid \dot{z}=X_{H}(t, z), z(0), z(1) \in L\right\} \text {. }
$$

We call any such element $z$ in $\operatorname{Chord}(H ; L, L))$ a Hamiltonian chord of $L$. This Cauchy-Riemann equation is called the dynamical version in [Oh3].

Equivalently one can also consider the genuine Cauchy-Riemann equation

$$
\left\{\begin{array}{l}
\frac{\partial v}{\partial \tau}+J^{H} \frac{\partial v}{\partial t}=0 \\
v(\tau, 0) \in \phi_{H}^{1}(L), v(\tau, 1) \in L
\end{array}\right.
$$


for the path $u: \mathbb{R} \rightarrow \mathcal{P}\left(\phi_{H}^{1}(L), L\right)$

$$
\mathcal{P}\left(\phi_{H}^{1}(L), L\right)=\left\{\gamma:[0,1] \rightarrow T^{*} N \mid \gamma(0) \in \phi_{H}^{1}(L), \gamma(1) \in L\right\}
$$

and $J_{t}^{H}=\left(\phi_{H}^{t}\left(\phi_{H}^{1}\right)^{-1}\right)_{*} J_{t}$. We note that whenever $\operatorname{supp} \phi_{H} \subset V, J_{t}^{H} \in$ $\mathcal{J}_{\omega}\left(V, J_{g}\right)$ for all $t \in[0,1]$. We call this version the geometric version. The upshot is that there is a filtration preserving isomorphisms between the dynamical version and the geometric version of the Lagrangian Floer theories.

We now describe the geometric version of the Floer homology in some more details. We denote by $\widetilde{\mathcal{M}}\left(L_{H}, L ; J^{H}\right)$ the set of finite energy solutions and $\mathcal{M}\left(L_{H}, L ; J^{H}\right)$ to be its quotient by $\mathbb{R}$-translations. In the unobstructed case [FOOO1], this gives rise to the geometric version of the Floer homology $H F_{*}\left(\phi_{H}^{1}(L), L, \widetilde{J}\right)$ of the type [Fl1] whose generators are the intersection points of $\phi_{H}^{1}(L) \cap L$. An advantage of this version is that it depends only on the Lagrangian submanifold $\left(\phi_{H}^{1}(L), L\right)$ depending loosely on $H$.

The following is a straightforward to check but is a crucial lemma.

Lemma 1.7. (1) The map $\Phi_{H}: \phi_{H}^{1}(L) \cap L \rightarrow \mathcal{C} h o r d(H ; L, L)$ defined by

$$
x \mapsto z_{x}^{H}(t)=\phi_{H}^{t}\left(\left(\phi_{H}^{1}\right)^{-1}(x)\right)
$$

gives rise to the one-one correspondence between the set $\phi_{H}^{1}(L) \cap L \subset$ $\mathcal{P}\left(\phi_{H}^{1}(L), L\right)$ as constant paths and the set of solutions of Hamilton's equation of $H$.

(2) The map $a \mapsto \Phi_{H}(a)$ also defines a one-one correspondence from the set of solutions of (4.1) and that of

$$
\left\{\begin{array}{l}
\frac{\partial v}{\partial \tau}+J^{H} \frac{\partial v}{\partial t}=0 \\
v(\tau, 0) \in \phi_{H}^{1}(L), v(\tau, 1) \in L
\end{array}\right.
$$

where $J^{H}=\left\{J_{t}^{H}\right\}, J_{t}^{H}:=\left(\phi_{H}^{t}\left(\phi_{H}^{1}\right)^{-1}\right)^{*} J_{t}$. Furthermore, (1.13) is regular if and only if (1.10) is regular.

Once we have transformed (1.10) to (1.13), we can further deform $J^{H}$ to the constant family $J_{0}$ inside $\mathcal{J}_{\omega}\left(V, J_{g}\right)$ and consider

$$
\left\{\begin{array}{l}
\frac{\partial v}{\partial \tau}+J_{0} \frac{\partial v}{\partial t}=0 \\
v(\tau, 0) \in \phi_{H}^{1}(L), v(\tau, 1) \in L
\end{array}\right.
$$

for each given $J_{0} \in \mathcal{J}_{\omega}$, a time-independent family. We will fix a generic $J_{0}$ in the rest of the paper and assume $L$ is transversal to $\phi_{H}^{1}(L)$ by considering a 
$C^{\infty}$-small perturbation of $H$ if necessary. This latter deformation preserves the filtration of the associated Floer complexes [Oh3]. A big advantage of considering this equation is that it enables us to study the behavior of spectral invariants for a sequence of $L_{i}$ converging to $L$ in Hausdorff distance.

\subsection{Statement of main results}

The following thick-thin dichotomy of the Floer moduli spaces is a crucial ingredient. This is a variation of Proposition $4.1[\mathrm{Oh} 2]$ in the $C^{0}$ context.

Theorem 1.8 (Compare with Proposition $4.1[\mathrm{Oh} 2]$ ). Let $L \subset(M, \omega)$ be a compact Lagrangian submanifold and let $V \subset \bar{V} \subset U$ be a pair of Darboux neighborhoods of $L$. Consider a $V$-engulfed Hamiltonian path $\phi_{H}$. Then whenever $\bar{d}\left(\phi_{H}^{1}, i d\right) \leq \delta$ for any $\delta<d(V, \Theta)$, any solution of $v$ of (1.14) satisfies one of the following alternatives:

(1) Either

$$
\text { Image } v \subset D_{\delta}(L) \subset V
$$

where $D_{\delta}(L)$ is the $\delta$-neighborhood of $L$.

(2) or Image $v \not \subset V$. In this case, we also have $\int v^{*} \omega \geq C\left(J_{0}, V\right)$ where $C\left(J_{0}, V\right)>0$ is a constant depending only on $\delta$ and $V$.

We call $v$ a thin trajectory if Image $v \subset V$ and a thick trajectory otherwise. We call a thin trajectory very thin if it satisfies (1.15) in addition. This theorem basically says that all thin trajectories are indeed very thin and all thick trajectories have area bounded below away from zero. The proof of this theorem is an easy application of maximum principle on the $J_{0}$-convex domain $V$ and the monotonicity formula for the $J_{0}$-holomorphic curves. We would like to emphasize that the meaning of thin trajectories here is different from that of [Oh2] (or the 'short' trajectories in [Che1]) in that they could have large areas unlike the case of latter.

This theorem enables us to define the local Floer homology in a welldefined way by counting thin trajectories. We denote this local Floer homology by

$$
H F_{*}^{[i d]}\left(\phi_{H}^{1}(L), L ; U\right), \quad \text { or } H F_{*}^{[i d]}(H,(L, L) ; U) .
$$

By definition, $H F^{[i d]}\left(\phi_{H}^{1}(L), L ; U\right)$ is always well-defined without any unobstructedness assumption of $L \subset M$ such as exactness or monotonicity of the pair $(L, M)$ or the unobstructedness in the sense of [FOOO1]. 
Once the above thick-thin dichotomy results of the Floer moduli spaces for the boundary map and for the chain map are established, essentially the same isolatedness argument as in [Oh2] gives rise to the following computation.

Theorem 1.9. Let $L \subset M$ be as above and $U$ be a Darboux neighborhood of $L$ and $\mathcal{H}: s \mapsto H(s)$ a family of $U$-engulfed Hamiltonians with $H(0)=$ 0 . Then if $\max _{s \in[0,1]} \bar{d}\left(\phi_{H(s)}^{1}, i d\right)<\delta$ and $\left|J_{t}-J_{0}\right|_{C^{1}}<\delta$ for some time independent $J_{0}$ and if $J$ is $\left(L, \phi_{H}^{1}(L)\right)$-regular, then

$$
H F_{*}(H, L ; J ; U) \cong H_{*}(L ; \mathbb{Z}) .
$$

We would like to emphasize that the presence of the engulfed homotopy $\mathcal{H}$ is crucial in the statement of this theorem, because the commonly used linear homotopy $s \mapsto s H$ may not be $U$-engulfed and so may not induce a chain map between the local Floer complex, even when $H_{0}, H_{1}$ are $U$-engulfed. In this regard, statement and the proof of this theorem given in section 5 may be the most novel points of the mathematics in the present article.

To perform the above computation, we need to study the behavior of the local Floer homology under the change of Hamiltonians. In this regard, we consider a 1-parameter family of Hamiltonians (or a 2-parameter family of functions on $M) \mathcal{H}=\{H(s)\}_{0 \leq s \leq 1}$ with $H(0) \equiv 0$ and

$$
\max _{s \in[0,1]} \bar{d}\left(\phi_{H(s)}^{1}, i d\right)<\delta
$$

for a sufficiently small $\delta=\delta_{0}\left(M, \omega ; J_{0}\right)$. We fix an elongation function $\rho$ : $\mathbb{R} \rightarrow[0,1]$ satisfying

$$
\begin{aligned}
& \rho(\tau)= \begin{cases}0 & \tau \leq 0 \\
1 & \tau \geq 1\end{cases} \\
& \rho^{\prime} \geq 0
\end{aligned}
$$

and define its dual $\widetilde{\rho}:=1-\rho$. We will consider the lemma in the Lagrangian setting over the path $s \mapsto H(s)$ for $\mathcal{H}=\{H(s)\}_{s \in[0,1]} \subset \mathcal{H}_{\delta}^{\text {engulf }}(M)$ with $H(0) \equiv 0$ for $\delta$ sufficiently small. Again the smallness will depend only on $(M, \omega)$.

We consider the Cauchy-Riemann equation with moving boundary condition

$$
\left\{\begin{array}{l}
\frac{\partial v}{\partial \tau}+J_{0} \frac{\partial v}{\partial t}=0 \\
v(\tau, 0) \in \phi_{H(\rho(\tau))}^{1}(L), v(\tau, 1) \in L
\end{array}\right.
$$


Then we prove the following analog to Theorem 1.8 for chain maps. This is the analogue of the handle sliding lemma from [Oh5, Oh8] which was studied for the case with $C^{2}$-smallness of Hamiltonians replaced by the smallness in hamiltonian topology (and also in the Lagrangian context).

Theorem 1.10 (Handle sliding lemma). Consider the path $\mathcal{H}: s \mapsto H(s)$ of engulfed Hamiltonians $H(s)$ satisfying (1.16) and fix an elongation function $\rho: \mathbb{R} \rightarrow[0,1]$. Then whenever $\bar{d}\left(\phi_{H(s)}^{1}, i d\right) \leq \delta<d(V, \Theta)$, any finite energy solution $v$ of (1.18) satisfies one of the following alternatives:

(1) Either

$$
\text { Image } v \subset D_{\delta}(L) \subset V \text {, }
$$

(2) or Image $v \not \subset V$. In this case, we also have $\int v^{*} \omega \geq C\left(J_{0}, V\right)$ where $C\left(J_{0}, V\right)>0$ is a constant depending only on $\delta$ and $V$.

Similarly as before done for the boundary map, we can transform a solution $v$ of (1.14) to that of the perturbed Cauchy-Riemann equation with fixed boundary condition

$$
\left\{\begin{array}{l}
\frac{\partial u}{\partial \tau}-X_{K(\rho(\tau))}(u)+J\left(\frac{\partial u}{\partial t}-X_{H(\rho(\tau))}(u)\right)=0 \\
\lim _{\tau \rightarrow-\infty} u(\tau)=z^{-}, \lim _{\tau \rightarrow \infty} u(\tau)=z^{+} .
\end{array}\right.
$$

The Floer chain map $h_{H^{\rho}}: C F_{*}\left(H^{0}\right) \rightarrow C F_{*}\left(H^{1}\right)$ can be defined by considering either the suitable moduli space of solutions (1.20) or that of (1.18).

Using the above constructed local Floer homology, we can assign the local spectral invariants which we denote by $\rho_{U}^{l a g}(H ; a)$ for $a \in H^{*}(L ; \mathbb{Z})$. We will restrict to the case $a=P D[M]=1$. To highlight the localness of the invariant we denote by $\rho_{U}^{\operatorname{lag}}\left(H ; 1_{0}\right)$ the corresponding invariant. We denote the global spectral invariant associated to 1 by $\rho^{l a g}(H ; 1)$ as in [Oh14].

By specializing to the case of zero section $o_{N}$ of $T^{*} N$, we can define the local Floer complex

$$
\left(C F_{*}\left(F ; U, T^{*} N\right), \partial_{U}\right)
$$

for any $F \in \mathcal{H}_{\delta}^{\text {engulf }}\left(T^{*} N\right)$ provided $\delta>0$ is sufficiently small.

When Theorem 1.8 and Theorem 1.10 are applied to the cotangent bundle $T^{*} L$, we obtain the following

Corollary 1.11. Consider a pair of open neighborhoods $V \subset \bar{V} \subset U$ of $o_{L}$ in $T^{*} L$ be given where $V$ is $J_{0}$-convex. Assume $\mathcal{H}=\{H(s)\}$ is an engulfed isotopy with $F=H(1)$ satisfying (1.16). Fix an elongation function $\rho$ : 
$\mathbb{R} \rightarrow[0,1]$ and consider the equation (1.18). Then there exists $\delta>0$ with $\delta<d(V, \theta)$ such that whenever

$$
\max _{s \in[0,1]} \bar{d}\left(\phi_{H(s)}^{1}, i d\right)<d(V, \Theta)
$$

the followings hold:

(1) For $F=H(1)$, any solution of $v$ of (1.14) with $\operatorname{Im} v \subset U$ is very thin.

(2) Fix an elongation function $\rho: \mathbb{R} \rightarrow[0,1]$ and consider the equation (1.18). Then any finite energy solution $v$ with $\operatorname{Im} v \subset U$ is thin (and so very thin).

When we specialize our construction, using Corollary 1.11 , to a $J_{0}$ convex neighborhood of the zero section in the cotangent bundle, we can define the local version $\rho_{V}^{l a g}\left(F ; 1_{0}\right)$ of the Lagrangian spectral invariants. We refer to section 6 for the detailed construction of this local invariant.

Once we have achieved localizations of various entities arising in Floer complex in the previous subsection, the following equality can be proven by the same argument used in the proof of Theorem 6.2 using the localized version of Lagrangian spectral invariants and basic phase function.

Theorem 1.12. Fix an open neighborhood $V \subset T^{*} L$ of $o_{L} \subset T^{*} L$ that is $J_{0}$-convex. Let $\mathcal{H}=\{H(s)\}$ be an engulfed isotopy with $H(0)=0$ and $H(1)=F$. Then for any $F \in \mathcal{H}_{\delta}^{\text {engulf }}(M ; V)$,

$$
\rho_{V}^{l a g}\left(F ; 1_{0}\right)=\rho^{l a g}(F ; 1) .
$$

Denote by $f_{F}^{V}$ the basic phase function defined by

$$
f_{F}^{V}(q)=\rho_{V}^{l a g}(F ;\{q\})
$$

where $\rho_{V}^{\operatorname{lag}}(F ;\{q\})$ is the spectral invariant defined by considering the local Floer complex $C F\left(L, T_{q}^{*} N ; V\right)$ instead of the global complex $C F\left(L, T_{q}^{*} N\right)$ on $T^{*} N$.

Theorem 1.13. Let $V \subset T^{*} N$ be as before. Then

$$
f_{F}^{V}=f_{F}
$$

for any $V$-engulfed $F$.

One important consequence of the above theorems is the inequalities

$$
\rho_{V}^{\operatorname{lag}}\left(F ; 1_{0}\right), \quad \max f_{H}^{V} \leq E^{-}(F) .
$$


Remark 1.14. We recall from [Oh3, Oh6] that the proof of the inequality $\rho^{\text {lag }}(F ; a) \leq E^{-}(F)$ therein is based on the computation of the action changes under the linear homotopy $s \mapsto s F$. This cannot be directly applied to the study of the local version of spectral invariants because the linear homotopy may not be $V$-engulfed as mentioned before.

In Appendix, we also localize the Hamiltonian Floer complex for a future purpose. We apply the above constructions to the graph

$$
\operatorname{Graph} \phi_{F}^{1}=\left\{\left(\phi_{H}^{1}(x), x\right) \mid x \in M\right\} \subset M \times M
$$

of engulfed Hamiltonian $F$ on $M$ satisfying

$$
\bar{d}\left(\phi_{F}^{1}, i d\right)<\delta
$$

for a sufficiently small $\delta>0$. We define

$$
\mathcal{H}_{\delta}^{\text {engulf }}(M) \subset C^{\infty}([0,1] \times M, \mathbb{R})
$$

to be the set of such Hamiltonian $F$ 's, and call the associated Hamiltonian path an engulfed Hamiltonian $C^{0}$-approximate loop.

Consider $\mathcal{U} \subset \mathcal{L}_{0}(M)$ defined by

$$
\mathcal{U}=\mathcal{U}\left(U_{\Delta}\right)=\left\{\gamma \in \mathcal{L}_{0}(M) \mid(\gamma(t), \gamma(0)) \in U_{\Delta}\right\} .
$$

We define the local Floer homology

$$
H F_{*}^{[i d]}(F, J ; \mathcal{U}), \quad \mathcal{U} \subset \mathcal{L}_{0}(M)
$$

by counting the 'thin' trajectories such that their images are contained in a neighborhood $\mathcal{U}$ of the set of constant paths in $M$. Again we would like to emphasize that we have not control of the area or filtration.

We denote by $\rho_{\mathcal{U}}^{\text {ham }}\left(\phi_{F} ; 1\right)=\rho_{\mathcal{U}}^{\text {ham }}(\underline{F} ; 1)$ the (local) spectral invariant associated to $1 \in H^{*}(L)$.

From now on, we will always assume that all the Hamiltonians in the rest of the paper are engulfed one way or the other, unless otherwise said explicitly.

We would like to thank D. McDuff and H. Hofer for pointing out a crucial gap in our $W^{1, p}$-precompactness proof in the previous version of the present paper. This forces us to abandon the area argument to obtain the thick-thin dichotomy of Floer moduli spaces but exploit the maximum principle instead to obtain a dichotomy result that will do our purpose of extracting the local 
Floer complex out of the global Floer complex. We also thank anonymous referees for many suggestions to improve presentation of the paper, and the suggestion of terminology 'engulfed' instead of 'engulfable', which suits much better for the current circumstance considered in the present paper.

\section{Local Floer chain module of engulfed Hamiltonian path $\phi_{H}$}

From now on, we will fix a pair of Darboux neighborhood $V \subset \bar{V} \subset U$ of $L$ in $M$ and assume $H$ is $V$-engulfed, i.e., satisfies

$$
\phi_{H^{t}}(L) \subset V \subset \bar{V} \subset U
$$

for all $t \in[0,1]$.

Next we recall the Lagrangian analogue of the Novikov $\operatorname{ring} \Gamma_{\omega}=\Gamma(M, \omega)$ from [FOOO1]. Denote by $I_{\omega}: \pi_{2}(M, L) \rightarrow \mathbb{R}$ the evaluations of symplectic area. We also define another integer-valued homomorphism $I_{\mu}: \pi_{2}(M, L) \rightarrow$ $\mathbb{Z}$ by

$$
I_{\mu}(\beta)=\mu\left(w^{*} T M,(\partial w)^{*} T L\right)
$$

which is the Maslov index of the bundle pair $\left(w^{*} T M,(\partial w)^{*} T L\right)$ for a (and so any) representative $w:\left(D^{2}, \partial D^{2}\right) \rightarrow(M, L)$ of $\beta$.

Definition 2.1. We define

$$
\Gamma_{(\omega, L)}=\frac{\pi_{2}(M, L)}{\operatorname{ker} I_{\omega} \cap \operatorname{ker} I_{\mu}}
$$

and $\Lambda(\omega, L)$ to be the associated Novikov ring.

We briefly recall the basic properties on the Novikov ring $\Lambda_{(\omega, L)}(R)$ where $R$ is a commutative ring where $R$ could be $\mathbb{Z}_{2}, \mathbb{Z}$ or $\mathbb{Q}$ for example. We will just use the letter $R$ for the coefficient ring which we do not specify. Basically $R$ will be $\mathbb{Q}$ when the associated moduli space is orientable as in the case of Graph $\phi_{H}^{1}$ for a Hamiltonian diffeomorphism $\phi_{H}^{1}$ which is of our main interest.

We put

$$
q^{\beta}=T^{\omega(\beta)} e^{\mu_{L}(\beta)},
$$

and

$$
\operatorname{deg}\left(q^{\beta}\right)=\mu_{L}(\beta), \quad E\left(q^{\beta}\right)=\omega(\beta)
$$


which makes $\Lambda_{(\omega, L)}$ and $\Lambda_{0,(\omega, L)}$ become a graded ring in general. We have the canonical valuation $\nu: \Lambda_{(\omega, L)} \rightarrow \mathbb{R}$ defined by

$$
\nu\left(\sum_{\beta} a_{\beta} T^{\omega(\beta)} e^{\mu_{L}(\beta)}\right)=\min \left\{\omega(\beta) \mid a_{\beta} \neq 0\right\} .
$$

It induces a valuation on $\Lambda_{(\omega, L)}$ which induces a natural filtration on it. This makes $\Lambda_{(\omega, L)}$ a filtered graded ring. For a general Lagrangian submanifold, this ring may not even be Noetherian but it is so if $L$ is rational, i.e., $\Gamma(L ; \omega)$ is discrete.

Now consider a nondegenerate $V$-engulfed Hamiltonian $H$ where $V$ is a given Darboux neighborhood of $L$. We denote by $\Omega(L, L)$ the set of paths $\gamma:[0,1]$ with $\gamma(0), \gamma(1) \in L$. In general $\Omega(L, L)$ is not connected but it has the distinguished component of constant paths, which we denote by

$$
\Omega_{0}(L, L) .
$$

When $H$ is $V$-engulfed, the path space $\Omega\left(\phi_{H}^{1}(L), L\right)$ also carries the distinguished component of the path $t \mapsto \phi_{H}^{t}(p)$ for $p \in L$. We denote by

$$
\Omega_{0}\left(\phi_{H}^{1}(L), L\right)
$$

the corresponding component. Then we denote by $\widetilde{\Omega}_{0}(L, L)$ the Novikov covering space and $\pi: \widetilde{\Omega}_{0}(L, L) \rightarrow \Omega_{0}(L, L)$ the projection. We denote by $[z, w]$ an element of $\widetilde{\Omega}_{0}(L, L)$.

Following [Che2] we say that two elements of Crit $\mathcal{A}_{H}$ are equivalent if they belong to the same connected component of the set

$$
\pi^{-1}\left(\left\{\gamma \in \Omega_{0}(L, L) \mid \gamma([0,1]) \subset U\right\}\right) \subset \widetilde{\Omega}_{0}(L, L) .
$$

Then the projection $\pi: \widetilde{\Omega}_{0}(L, L) \rightarrow \Omega_{0}(L, L)$ bijectively maps each equivalence class of Crit $\mathcal{A}_{H}$ to $\mathcal{C}$ hord $(H ; L, L)$. In the current case of $V$-engulfed Hamiltonian, there is a 'canonical equivalence class' represented by the pairs $\left[z, w_{z}\right]$ for each given chord $z \in \mathcal{C} h o r d(H ; L, L)$, where $w_{z}$ is the (homotopically) unique cone-contraction of $z$ to a point in $L$. We denote this equivalence class by $\operatorname{Crit}^{[i d]} \mathcal{A}_{H} \subset \operatorname{Crit} \mathcal{A}_{H}$. This provides a canonical section of $\pi: \widetilde{\Omega}_{0}(L, L) \rightarrow \Omega_{0}(L, L)$ when restricted to $\operatorname{Chord}(H ; L, L) \subset \Omega_{0}(L, L)$. This in turn induces a natural $\Gamma_{(\omega, L)}$-action on Crit $\mathcal{A}_{H}$ which gives rise to the bijection

$$
\mathrm{Crit}^{[i d]} \mathcal{A}_{H} \times \Gamma_{(\omega, L)} \rightarrow \operatorname{Crit} \mathcal{A}_{H} .
$$


Remark 2.2. Note that for any $[z, w] \in \mathrm{Crit}^{[i d]} \mathcal{A}_{H},(2.1)$ implies $z(t) \in V$ since $z(0) \in \phi_{H}^{1}(L)$. Therefore the action value $\mathcal{A}_{H}([z, w])$ will not change even if we cut-off $H$ outside $V$.

We denote

$$
\mathrm{Crit}^{[g]} \mathcal{A}_{H}=g \cdot \mathrm{Crit}^{[i d]} \mathcal{A}_{H}, \quad g \in \Gamma_{(\omega, L)} .
$$

Then we denote their associated $R$-module by

$$
C F_{*}^{[g]}((L, L), H ; U), \quad C F_{*}^{[i d]}((L, L), H ; U)=C F_{*}^{[i d]}((L, L), H ; U) .
$$

We want to remark that $C F_{*}^{[i d]}((L, L), H ; U)$ coincides with the local Floer complex that was used by the author in [Oh2] for the $C^{2}$-small cases.

The above discussion in turn gives rise to the isomorphism

$$
C F^{[g]}((L, L), H ; U) \otimes_{R} \Lambda_{(\omega, L)} \cong C F_{*}((L, L) ; H)
$$

as a $\Lambda_{(\omega, L)}$-module for each $g \in \Lambda_{(\omega, L)}$. Following [Che1, Che2], we denote

$$
\operatorname{leng}(u):=E_{J}(u)=E_{J_{0}}(v)=\operatorname{area}(v) .
$$

Now we note that the Floer (pre)-boundary map

$$
\partial: C F_{*}((L, L) ; H) \rightarrow C F_{*}((L, L) ; H)
$$

is $\Lambda_{(\omega, L)}$-equivariant and has the decomposition

$$
\partial=\sum_{\lambda \in \mathbb{R} \geq 0} \partial_{\lambda}
$$

where $\partial_{\lambda}$ is the contribution arising from $u \in \mathcal{M}(L, L ; H)$ with

$$
\text { leng }(u)=\lambda>0 .
$$

\section{Thick-thin dichotomy of Floer trajectories}

This section is a modification of section 3 of [Oh2] which treats the case of $C^{2}$-small perturbation of Hamiltonians $H$. In this section, we will replace the condition of $\phi_{H}$ being $C^{1}$-small by that of $\phi_{H}$ being $C^{0}$-small.

Consider a sequence $v: \mathbb{R} \times[0,1] \rightarrow M$ of solutions of (1.14) associated to $H$ and $J_{0}$. We re-state Theorem 1.8 here. 
Theorem 3.1. Let $L \subset(M, \omega)$ be a compact Lagrangian submanifold and let $V \subset \bar{V} \subset U$ be a pair of Darboux neighborhoods of L. Consider a $V$ engulfed Hamiltonian path $\phi_{H}$. Then there exists $\delta>0$ depending only on $\varepsilon$ (and $(M, \omega)$ ) such that whenever $\bar{d}\left(\phi_{H}^{1}, i d\right) \leq \delta$, any solution of $v$ of $(1.14)$ satisfies one of the following alternatives:

(1) Image $v \subset V$ and $\max d\left(v(z), o_{L}\right) \leq d_{\mathrm{H}}\left(\phi_{H}^{1}(L), L\right)$,

(2) Image $v \not \subset V$ and $\int v^{*} \omega \geq C\left(J_{0}, V\right)$ where $C\left(J_{0}, V\right)>0$ is a constant depending only on $V$.

Proof. Suppose that Image $v \not \subset V$. Then there exists a point $v(z) \notin V$ and so

$$
d\left(v(z), v(\partial(\mathbb{R} \times[0,1])) \geq \min \left\{d_{\mathrm{H}}\left(\partial V, \phi^{1}(L)\right), d_{\mathrm{H}}\left(\partial V, o_{L}\right)\right\} .\right.
$$

Then the monotonicity formula implies

$$
\int v^{*} \omega \geq C^{\prime} \cdot\left(\min \left\{d_{\mathrm{H}}\left(\partial V, \phi^{1}(L)\right), d_{\mathrm{H}}\left(\partial V, o_{L}\right)\right\}\right)^{2}
$$

where $C^{\prime}$ is the monotonicity constant of $\left(M, \omega, J_{0}\right)$ in the monotonicity formula. Considering $\delta<\frac{1}{4} \cdot d_{\mathrm{H}}\left(\partial V, o_{L}\right)$ and setting

$$
C\left(J_{0}, V\right):=\frac{1}{2} C^{\prime} \cdot\left(d_{\mathrm{H}}\left(\partial V, o_{L}\right)-\delta\right)^{2} \geq \frac{1}{4} C^{\prime} d_{\mathrm{H}}\left(\partial V, o_{L}\right)^{2}
$$

(2) follows.

For the curve $v$ of the type (1), the maximum principle applied to $J_{0^{-}}$ holomorphic curves contained in the Darboux neighborhood, we obtain the maximum distance of $v(z)$ from $L$ is achieved on the boundary $\mathbb{R} \times\{0,1\}$. But by the boundary condition, we have

$$
\left.\max _{z \in \mathbb{R} \times\{0,1\}} d\left(v(z), o_{L}\right) \leq d_{\mathrm{H}}\left(\phi_{H}^{1}(L), L\right)\right) .
$$

This finishes the proof.

Remark 3.2. We would like to note that the area property for the trajectories $v$ of the type (2) spelled out as

$$
\int v^{*} \omega \geq C\left(J_{0}, V\right)
$$

with constant $C\left(J_{0}, V\right)>0$ independent of $H$ will not be used in this paper. 
We now decompose $\partial$ into

$$
\partial=\partial_{(0)}+\partial^{\prime}
$$

where $\partial_{(0)}$ is the sum of contribution of thin trajectories and $\partial^{\prime}$ that of thick trajectories.

Remark 3.3. We would like to emphasize that even when $H$ is $C^{1}$-small this decomposition does not respect the one given in (2.3). This is a contrast from the case of $C^{2}$-small $H$ : in that case it was proven in [Oh2] that there is a constant $\delta_{0}(H)$ satisfying $\delta(H) \rightarrow 0$ as $\|H\|_{C^{2}} \rightarrow 0$ such that all thin trajectories have area less than $\delta_{0}(H)$ and that all thick trajectories have area greater than $\frac{1}{2} A\left(M, L, J_{0}\right)$ and hence

$$
\partial_{(0)}=\sum_{|\lambda|<\delta_{0}(H)} \partial_{\lambda}, \quad \partial^{\prime}=\sum_{\lambda>\frac{1}{2} A\left(M, L, J_{0}\right)} \partial_{\lambda} .
$$

We denote $u \in \operatorname{supp} \partial$, supp $\partial_{(0)}$, and supp $\partial^{\prime}$ respectively, if the map $u$ nontrivially contributes to the corresponding operators.

Definition 3.4. We call $\left(C F_{*}^{[i d]}((L, L), H ; U), \partial_{U}\right)$ the local Floer complex of $H$ in $U$ which is defined to be

$$
\begin{aligned}
C F_{*}^{[i d]}((L, L), H ; U) & =R \cdot\left\{\mathrm{Crit}^{[i d]} \mathcal{A}_{H}\right\}, \\
\partial_{U} & =\left.\partial_{(0)}\right|_{C F_{*}^{[i d]}((L, L), H ; U)}
\end{aligned}
$$

The $\Lambda_{(\omega, L)}$-equivariance of $\partial$ gives rise to

$$
\left.\widehat{g} \circ \partial_{(0)}\right|_{C F_{*}^{[i d]}((L, L), H ; U)}=\left.\partial_{(0)}\right|_{C F_{*}^{[g]}((L, L), H ; U)} \circ \widehat{g}
$$

and $\widehat{g}$ carries a natural weight given by

$$
\mathcal{A}_{F}(g \cdot[z, w])-\mathcal{A}_{F}([z, w]),[z, w] \in \operatorname{Crit} \mathcal{A}_{F}
$$

which does not depend on the choice of $[z, w] \in \operatorname{Crit} \mathcal{A}_{F}$. In fact this real weight is nothing but the value $\omega([g])$.

Proposition 3.5. Let $\delta>0$ where $\delta$ is the constant given in Theorem 3.1. Then $\partial_{U}^{2}=0$ and so the local Floer homology

$$
H F_{*}^{[i d]}((L, L), H ; U)=\operatorname{ker} \partial_{U} / \operatorname{im} \partial_{U}
$$

is well-defined. 
Proof. Since all the thin trajectories have their image contained in the Darboux neighborhood $U$, concatenations of thin trajectories are also thin and the thin part of Floer moduli spaces for the pair $\left(\phi_{H}^{1}(L), L\right)$ does not bubbleoff. Then the standard compactness and gluing argument immediately finishes the proof.

In the next section we will compute the group $H F_{*}^{[i d]}((L, L), F ; U)$, when $F=H(1)$ for a 2-parameter family $\mathcal{H}=\{H(s)\}_{s \in[0,1]}$ with $H(0)=0$ and $H(s) \in \mathcal{H}_{\delta}^{\text {engulf }}(M)$. We denote by

$$
\bar{d}\left(\phi_{\mathcal{H}}^{1}, i d\right):=\max _{s \in[0,1]} \bar{d}\left(\phi_{H(s)}^{1}, i d\right)
$$

the $C^{0}$-distance of $\mathcal{H}$ to the constant family $i d$.

\section{Handle sliding lemma for engulfed isotopy of $C^{0}$-approximate loops}

In this section, we examine another important element in the chain level theory, the handle sliding lemma introduced in [Oh5] for the Hamiltonian $H$ that is sufficiently $C^{2}$-small. We will consider the lemma in the Lagrangian setting over the path $s \mapsto H(s)$ for $\mathcal{H}=\{H(s)\}_{s \in[0,1]} \subset \mathcal{H}_{\delta}^{\text {engulf }}(M)$ with $H(0) \equiv 0$ for $\delta$ sufficiently small. Again the smallness will depend only on $(M, \omega)$.

For a family $\mathcal{H}=\{H(s)\}_{s \in[0,1]}$, we also study the comparison of this equation with the moving boundary condition. For such a family, we consider the geometric version first

$$
\left\{\begin{array}{l}
\frac{\partial v}{\partial \tau}+J_{0} \frac{\partial v}{\partial t}=0 \\
v(\tau, 0) \in \phi_{H(\rho(\tau))}^{1}(L), v(\tau, 1) \in L
\end{array}\right.
$$

for the path $v: \mathbb{R} \times[0,1] \rightarrow M$. If we define a map $u: \mathbb{R} \times[0,1] \rightarrow M$

$$
u(\tau, t)=\phi_{H(\rho(\tau))}^{t}\left(\phi_{H(\rho(\tau))}^{1}\right)^{-1}(v(\tau, t)),
$$

A simple calculation proves that $u$ satisfies $u(\tau, 0), u(\tau, 1) \in L$ and

$$
\left\{\begin{array}{l}
\frac{\partial u}{\partial \tau}-X_{K(\rho(\tau))}(u)+J\left(\frac{\partial u}{\partial t}-X_{H(\rho(\tau))}(u)\right)=0 \\
u(\tau, 0), u(\tau, 1) \in L
\end{array}\right.
$$


where $K$ is the $s$-Hamiltonian generating the Hamiltonian vector field

$$
X_{K}(s, t, x):=\frac{\partial \phi}{\partial s}\left(\phi^{-1}(s, t, x)\right)
$$

of the 2-parameter family $(s, t) \mapsto \phi(s, t)=\phi_{H(s)}^{t} \phi_{H(s)}^{-1}$ and $J=J(s, t)=$ $(\phi(s, t))_{*} J_{0}$. We would like to highlight the presence of the terms $X_{K(\rho(\tau))}(u)$ in the above equation for $u$ and the definition of energy of $u$. The associated off-shell energy of (4.2) is given by

$$
E_{(H, K), J ; \rho}(u)=\frac{1}{2} \int_{-\infty}^{\infty} \int_{0}^{1}\left|\frac{\partial u}{\partial \tau}-X_{K(\rho(\tau))}(u)\right|_{J}+\left|\frac{\partial u}{\partial t}-X_{H(\rho(\tau))}(u)\right|_{J}^{2} d t d \tau .
$$

which coincides with

$$
\int_{-\infty}^{\infty} \int_{0}^{1}\left|\frac{\partial u}{\partial t}-X_{H(\rho(\tau))}(u)\right|_{J}^{2} d t d \tau
$$

on shell. The proof of the on-shell identities

$$
\int v^{*} \omega=E_{J_{0}}(v)=E_{(H, K), J ; \rho}(u)
$$

is straightforward and so omitted. With these correspondences, we have the obvious analog to Lemma 1.7 for the moving boundary condition, whose precise statement we omit.

Here we re-state Theorem 1.10 and give its proof here.

Theorem 4.1 (Handle sliding lemma). Consider the path $\mathcal{H}: s \mapsto H(s)$ of engulfed Hamiltonians $H(s)$ satisfying (1.16) and fix an elongation function $\rho: \mathbb{R} \rightarrow[0,1]$. Then there exists $\delta>0$ such that if $\bar{d}\left(\phi_{H(s)}^{1}, i d\right)<\delta<$ $d(V, \Theta)$, any finite energy solution $v$ of (1.18) satisfies one of the following alternatives:

(1) if Image $v \subset V, \max _{z} d\left(v(z), o_{L}\right) \leq \bar{d}\left(\phi_{H(s)}^{1}, i d\right)$,

(2) if Image $v \not \subset V, \int v^{*} \omega \geq C\left(J_{0}, V\right)$ where $C\left(J_{0}, V\right)>0$ is a constant depending only on $J_{0}$ and $V$.

Proof. The proof is the same as that of Theorem 3.1 and so omitted.

Now Theorem 4.1 together with this dichotomy of thick-thin trajectories enable us to decompose the Floer-Piunikhin (pre)-chain map

$$
\Psi_{\mathcal{H}}: C_{*}(L) \rightarrow C F_{*}((L, L), H(1) ; U)
$$


into the thick-thin decomposition

$$
\Psi_{\mathcal{H}}=\Psi_{\mathcal{H},(0)}+\Psi_{\mathcal{H}}^{\prime}
$$

similar to (3.1). Again it follows from Theorem 1.10 that those $v$ 's contributing non-trivially to $\Psi_{\mathcal{H},(0)}$ are very thin (and those contributing to $\Psi_{\mathcal{H}}^{\prime}$ has area bigger than $C\left(J_{0}, V\right)$.)

We refer to section 5.3 [FOOO1] or section 5 [FOOO3] for the details of the construction of the Floer-Piunikhin (pre)-chain map $\Psi_{\mathcal{H}}$.

Remark 4.2. The above Handle sliding lemma can be also proved by the same argument for the Floer chain map between $f$ and $H(1) \# f$ when $|f|_{C^{2}}$ is sufficiently small relative to $C\left(V, J_{0}\right)$. This way one can avoid using the Bott-Morse version of Floer chain map, the Floer-Piunikhin (pre)-chain map $\Psi_{\mathcal{H}}$.

\section{Computation of local Floer homology $H_{*}^{[i d]}((L, L), H ; U)$}

The role of the $C^{2}$-smallness in the construction of local Floer complex

$$
H F_{*}^{[i d]}((L, L), H ; U)
$$

in [Oh2] was two-fold. One is to make its flow $\phi_{H} C^{1}$-small which gives rise to a thick-thin operators of Floer operators. The other is for the construction of (local) chain isomorphism between the singular complex of $L$ and the Floer complex $C F_{*}^{[i d]}((L, L), H ; U)$ for which one needs to avoid bubbling (especially disc-bubbling) to ensure the chain isomorphism property of the Floer-Piunikhin's continuation map. For the latter purpose, we need to obtain some estimates of the filtration change for the Floer chain map between the identity path and $\phi_{H}$ over the family

$$
\mathcal{H}: s \mapsto H(s), \quad s \in[0,1] .
$$

In the present context, we do not have such control over the filtration change under the chain map we construct, even if one uses the adiabatic chain map mentioned before: Since we do not have any restriction on the $C^{2}$-norm of $H$, we will not have much control on the mesh of the partitions we make for the given approximating sequence $H_{i}$. To overcome this lack of control of the filtration, we use Conley and Floer's idea of continuation of maximal invariant sets [Co, Fl2, Oh2], which we now briefly summarize leaving more details thereto. 
We denote by $\mathcal{M}_{1}\left(J,\left(L^{\prime}, L\right) ; U\right)$ the set of pairs $(u, z)$ of $J$-holomorphic strips $u$ attached to the pair $\left(L^{\prime}, L\right)$ whose image is contained in $U$ and a marked point $z \in \mathbb{R} \times[0,1]$. We then denote

$$
\mathcal{S}\left(J,\left(L^{\prime}, L\right) ; U\right):=\overline{e v\left(\mathcal{M}_{1}\left(J,\left(L^{\prime}, L\right) ; U\right)\right)}
$$

and call it the maximal invariant set of the Cauchy-Riemann flow. For a given one parametric family

$$
\left(J^{\text {para }}, H^{\text {para }}\right) \in \operatorname{Map}\left([0,1]^{2}, \mathcal{J}_{\omega}\right) \times C^{\infty}\left([0,1]^{2} \times M, \mathbb{R}\right)
$$

with $H^{\text {para }}=\mathcal{H}$ with $H(0)=0$, we define a continuation $U^{\text {para }}$ between the maximal invariant sets $\mathcal{S}_{0} \subset U^{0}$ and $\mathcal{S}_{1} \subset U^{1}$ to be an open subset of $[0,1] \times M$ that satisfies

(1) For each $s \in[0,1]$ and all $t \in[0,1]$,

$$
L^{s} \subset U^{s}:=\left\{x \in M \mid(x, s) \in U^{\text {para }}\right\} .
$$

$(2)$

$$
\mathcal{S}_{s}:=\mathcal{S}\left(J^{s},\left(L^{s}, L\right) ; U^{s}\right)
$$

is isolated in $U^{s}$ for all $s \in[0,1]$.

The following isolatedness is a crucial ingredient in the construction of the isomorphism

$$
\left.H_{*}(L) \cong H F^{[i d]}\left(\phi_{H}^{1}(L), L\right), J ; U\right) .
$$

Proposition 5.1. $\mathcal{S}\left(J_{0},\left(\phi_{H(s)}^{1}(L), L\right) ; V\right)$ is isolated in $V$.

Proof. Consider the family $\mathcal{S}_{s}:=\mathcal{S}\left(J_{0},\left(\phi_{H(s)}^{1}(L), L\right) ; V\right)$ for $0 \leq s \leq 1$. Clearly $\mathcal{X}_{0}=\mathcal{S}\left(J_{0},(L, L) ; V\right)$ is isolated in $V$. Furthermore the isolatedness is an open property. Let $0<s_{0} \leq 1$ be the smallest $s$ at which $\mathcal{S}_{s_{0}}$ fails to be isolated in $V$. Then there exists some $z_{0}=\left(\tau_{0}, t_{0}\right) \in \mathbb{R} \times[0,1]$ such that $v\left(\tau_{0}, t_{0}\right) \in \partial \bar{V}$. Since $v(\tau, 0) \in \phi_{H(s)}^{1}(L)$ and $v(\tau, 1) \in L$ and $\phi_{H(s)}^{1}(L), L \subset$ $V$, this violates the maximum principle applied to the $J_{0}$-convex boundary of $V$. This finishes the proof.

Once we have set up these definitions and isolatedness, it immediately gives rise to the following theorem

Theorem 5.2. Suppose $\left(L^{\prime}, L ; J ; U\right)$ is as above. Suppose $H \in \mathcal{H}_{\delta}^{\text {engulf }}(L ; U)$ for a sufficiently small $\delta=\delta(\varepsilon)>0$. Then for any small perturbation $J^{\prime}$ of $J$ for which $\mathcal{M}\left(L^{\prime}, L ; J^{\prime} ; U\right)$ is Fredholm regular, 
(1) the homomorphism

$$
\partial_{U}: C F\left(L^{\prime}, L ; J^{\prime} ; U\right) \rightarrow C F\left(L^{\prime}, L ; J^{\prime} ; U\right), \quad \partial_{U} x=\sum_{y \in L \cap \phi_{H}^{1}(L)}\left\langle\partial_{U} x, y\right\rangle y
$$

satisfies $\partial_{U} \circ \partial_{U}=0$.

(2) And the corresponding quotients

$$
H F\left(L, L ;\left(H, J^{\prime}\right) ; U\right) \cong H F^{*}\left(L^{\prime}, L ; J^{\prime} ; U\right)=\operatorname{ker} \partial_{U} / \operatorname{im} \partial_{U}
$$

are isomorphic under the continuation $\left(\mathcal{S}^{\text {para }}, J^{\text {para }}, H^{\text {para }}, U^{\text {para }}\right)$ as long as the continuation is Floer-regular at the ends $s=0,1$.

After we establish this continuation invariance, we can apply it to the family $\mathcal{H}$ with $H(0)=0$ and prove the following theorem. The proof of this theorem together with that of Corollary 6.3 may be the most novel part of the mathematics of the present paper beyond those already established in [Oh2], [Oh3].

Theorem 5.3. Consider $\mathcal{H}=\{H(s)\} \subset \mathcal{H}_{\delta}^{\text {engulf }}(M)$ with $H(0)=0$. Then whenever $0<\delta<d(V, \Theta)$,

$$
H F^{[i d]}\left(\phi_{H}^{1}(L), L ; J^{\prime} ; U\right) \cong H_{*}(L ; \mathbb{Z})
$$

for any $J^{\prime}$ sufficiently close to $J_{0}$ in $C^{\infty}$-topology.

Proof. We consider the homotopy

$$
\mathcal{H}: s \mapsto H(s)
$$

and its reversal. Using the isolatedness of thin trajectories in Theorem 3.1 and Theorem 4.1, we define the local Floer-Piunikhin (pre)-chain maps

$$
\begin{aligned}
\Psi_{\mathcal{H}^{\rho},(0)}: C F_{*}^{[i d]}((L, L), 0 ; U) & \rightarrow C F_{*}^{[i d]}((L, L), H ; U), \\
\Phi_{\mathcal{H}^{\tilde{\rho}},(0)}: C F_{*}^{[i d]}((L, L), H ; U) & \rightarrow C F_{*}^{[i d]}((L, L), 0 ; U)
\end{aligned}
$$

and their compositions

$$
\begin{aligned}
\Psi_{\mathcal{H}^{\rho},(0)} \circ \Phi_{\mathcal{H}^{\tilde{\rho}},(0)}: C F_{*}((L, L), 0 ; U) & \rightarrow C F_{*}((L, L), H ; U), \\
\Phi_{\mathcal{H}^{\tilde{\rho}},(0)} \circ \Psi_{\mathcal{H}^{\rho},(0)}: C F_{*}((L, L), H ; U) & \rightarrow C F_{*}((L, L), 0 ; U) .
\end{aligned}
$$

Theorem 3.1 and Theorem 4.1 imply that all the above maps properly restrict to the maps between $C F_{*}^{[i d]}(0 ; U) \cong\left(C_{*}(L), \partial_{(0)}\right)$, the singular chain 
complex, and $C F_{*}^{[i d]}(H ; U)$ by isolating the thin trajectories. Since the thin trajectories cannot bubble-off, all these maps become chain maps between them. Therefore $\Psi_{\mathcal{H}^{\rho},(0)}$ and $\Phi_{\mathcal{H}^{\widetilde{\rho}},(0)}$ induce the isomorphisms between $H F_{*}^{[i d]}((L, L), 0 ; U) \cong H_{*}(L)$ and $H F_{*}^{[i d]}((L, L), H ; U)$ which are inverses to each other. More precisely, there exist a chain homotopy maps between $\Psi_{\mathcal{H}^{\rho},(0)} \circ \Phi_{\mathcal{H}^{\tilde{\rho}},(0)}$ and $i d_{C_{*}(L)}$, and $\Phi_{\mathcal{H}^{\tilde{\rho}},(0)} \circ \Psi_{\mathcal{H}^{\rho},(0)}$ and $i d_{C F_{*}(H)}$ respectively. (See [Oh1, FOOO3] for the proof of existence of such a chain homotopy.) Once this is established, we can compute $H F_{*}^{[i d]}((L, L), 0 ; U)$ inside the cotangent bundle $T^{*} L$. Then the arguments used in [Fl3] and [Oh2] prove the theorem.

This finishes the proof.

Remark 5.4. In the above proof, we would like to emphasize that no bubbling-off for the thin trajectories holds not because the area of a bubble will be big but because a bubble must go out of the given Darboux neighborhood of $L$ and hence cannot be thin. As we mentioned before we recall that thin trajectories could have large area.

\section{Localization on the cotangent bundle}

The main purpose of this section is to use the local Floer complex constructed on the cotangent bundle and localize the construction of Lagrangian spectral invariants introduced in [Oh3] which has been further studied in [Oh14].

We will also localize the triangle product similarly and the basic phase function in the current context of approximations of engulfed topological Hamiltonian loops in Appendix, for a future purpose.

\subsection{Localization of Lagrangian spectral invariants $\rho^{\text {lag }}(H ; 1)$}

We first specialize the general definition of spectral invariants $\rho^{\operatorname{lag}}(F ; 1)$ and $\rho_{V}^{l a g}\left(F ; 1_{0}\right)$ to the cotangent bundle. In this case of the Hamiltonian deformations of the zero section in the cotangent bundle, we do not need to use the Novikov ring but only use the coefficient ring $R$ and have only to use the single valued classical action functional

$$
\mathcal{A}_{F}^{c l}(\gamma)=\int \gamma^{*} \theta-\int_{0}^{1} F(t, \gamma(t)) d t
$$

in the evaluation of the level of the chains. 
We now assume that $L$ is connected. Using the isomorphism

$$
\left(\Psi_{\mathcal{H}}\right)_{*}: H_{*}\left(L, \Lambda_{(\omega, L)}\right) \rightarrow H F_{*}(F)
$$

where $\Psi_{\mathcal{H}}$ is the chain map defined in section 4 , we define

$$
\rho^{l a g}(F ; 1)=\inf _{\alpha \in\left(\Psi_{\mathcal{H}}\right)_{*}([L])} \lambda_{F}(\alpha)
$$

which is also the same as

$$
\inf _{\lambda}\left\{H F_{*}^{[i d], \lambda}(F) \neq 0\right\} .
$$

This is because $H F_{n}(F ; V)$ or $H F_{n}(F)$ has rank one and so all isomorphisms $H_{*}(L) \rightarrow H F_{*}(F)$ maps the fundamental cycle $[L]$ of $L$ to the same image modulo a non-zero scalar multiple and so the associated spectral invariants coincide (Confomality Axiom [Oh6]). Similarly we define

$$
\rho_{V}^{l a g}(F ; 1)=\inf _{\alpha \in\left(\Psi_{\mathcal{H},(0)}\right)_{*}([L])} \lambda_{F}(\alpha)
$$

which is also the same as

$$
\inf _{\lambda}\left\{H F_{*}^{[i d], \lambda}(F ; V) \neq 0\right\} .
$$

Remark 6.1. We would like to mention that the homomorphism $\left(\Psi_{\mathcal{H}}\right)_{*}$ and $\left(\Psi_{\mathcal{H},(0)}\right)_{*}$ do not depend on the choice of homotopy $\mathcal{H}$. But for the case $\left(\Psi_{\mathcal{H},(0)}\right)_{*}$ the whole family of Hamiltonians $H(s)$ for $s \in[0,1]$ should be assumed to be $V$-engulfed. For example, the commonly used the linear homotopy $s \mapsto s F$ may not be $V$-engulfed even when $F$ is $V$-engulfed. Because of this, the linear homotopy cannot be used to construct the local chain map in general. Here is one place where the presence of the engulfed family $\mathcal{H}$ of Hamiltonians is used in the definition of local Lagrangian spectral invariants.

Now we prove the following coincidence theorem of global and local spectral invariants.

Theorem 6.2. Let $\mathcal{H}=\{H(s)\}$ be a $V$-engulfed isotopy with $H(0)=0$ and $F=H(1)$. Then we have

$$
\rho_{V}^{l a g}\left(F ; 1_{0}\right)=\rho^{l a g}(F ; 1)
$$


Proof. For the given family

$$
\mathcal{H}: s \mapsto H(s), \quad s \in[0,1]
$$

we consider the continuation of maximal invariant sets defined in section 6 . For given one parametric family

$$
\left(J^{\text {para }}, H^{\text {para }}\right) \in M a p\left([0,1]^{2}, \mathcal{J}_{\omega}\right) \times C^{\infty}\left([0,1]^{2} \times M, \mathbb{R}\right)
$$

with $H^{\text {para }}=\mathcal{H}$ with $H(0)=0$ and $J^{\text {para }}=J_{0}$, all the Floer trajectories contributing to these maximal invariant sets are thin and so become very thin. In particular, the maximal invariant sets $\mathcal{S}_{s}$ are all contained in the given neighborhood $[0,1] \times D^{\delta}\left(T^{*} L\right)$ for all of $[0,1] \times M$ and $\mathcal{S}_{0}=L$.

This implies that the local Floer complex $\left(C F_{*}^{[i d]}(F), \partial_{(0)}\right)$ and the global one $\left(C F_{*}(F), \partial\right)$ define the same complex and also satisfies

$$
\left(\Psi_{\mathcal{H}}\right)_{*}([L])=\left(\Psi_{\mathcal{H},(0)}\right)_{*}([L])
$$

under the identification, provided $F$ is connected to 0 via an engulfed Hamiltonian homotopy $\mathcal{H}=\{H(s)\}$ is given. This finishes the proof.

The proof of the following corollary requires some care unlike the case of global Floer homology because the standard linear homotopy $s \mapsto s F$ may not be $V$-engulfed.

Corollary 6.3. For any $F \in \mathcal{H}_{\delta}^{\text {engulf }}\left(T^{*} L ; V\right), \rho_{V}^{\operatorname{lag}}\left(F ; 1_{0}\right) \leq E^{-}(F)$.

Proof. Knowing that Theorem 6.2 holds, we can consider the linear homotopy $s \mapsto s F$ and denote by $\Psi_{F}^{\text {lin }}$ the associated Floer-Piunikhin chain map $C_{*}(L) \rightarrow C F_{*}(F)$ for the global Floer complex instead. Then it is standard that $\Psi_{F}^{l i n}$ also induces an isomorphism $H_{*}(L) \rightarrow H F_{*}(F)$ in global Floer homology. More specifically we have

$$
\left(\Psi_{F}^{l i n}\right)_{*}([L])=\left(\Psi_{\mathcal{H}}\right)_{*}([L]) .
$$

(We emphasize that the corresponding cycles $\left(\Psi_{F}^{l i n}\right)_{\#}([L]),\left(\Psi_{\mathcal{H}}\right)_{\#}([L])$ are different in general. For example, the general estimate of the level of the cycle $\left(\Psi_{\mathcal{H}}\right)_{\#}([L])$ involve the derivative $\frac{\partial H(s)}{\partial s}$ which is uncontrolled in the topological Hamiltonian homotopy.) Using the cycle $\left(\Psi_{F}^{l i n}\right)_{\#}([L])$, it is easy to obtain the upper bound $\rho(F ; 1) \leq E^{-}(F)$ by the standard calculations. (See [Oh3, Oh5, Oh6] for example). This together with Theorem 6.2 gives rise to the proof. 
Remark 6.4. We would like to emphasize that unlike the isotopy $\mathcal{H}=$ $\{H(s)\}$ with $H(s) \in \mathcal{H}_{\delta}^{\text {engulf }}(L ; V)$, the isotopy of the time-one maps $\phi_{s F}^{1}$ for the linear isotopy $\mathcal{H}^{\text {lin }}: s \mapsto s F$ with $F=H(1)$ may not be uniformly $C^{0}$-small and hence the associated Floer trajectories of the chain map moduli space could go out of the neighborhood $V$. Because of this, the linear isotopy cannot be used to define a chain map from $C_{*}(L)$ to the local Floer complex $C F_{*}^{[i d]}(F ; V)$ and so the inequality stated in this corollary does not follow from by now the standard computation used in $[\mathrm{Oh} 6]$ to prove $\rho^{\operatorname{lag}}\left(F ; 1_{0}\right) \leq$ $E^{-}(F)$ for the global invariant.

\subsection{Localization of the basic phase function}

We consider the Lagrangian pair

$$
\left(o_{N}, T_{q}^{*} N\right), \quad q \in N
$$

and its associated Floer complex $C F\left(H ; o_{N}, T_{q}^{*} N\right)$ generated by the Hamiltonian trajectory $z:[0,1] \rightarrow T^{*} N$ satisfying

$$
\dot{z}=X_{H}(t, z(t)), \quad z(0) \in o_{N}, z(1) \in T_{q}^{*} N .
$$

Denote by $\operatorname{Chord}\left(H ; o_{N}, T_{q}^{*} N\right)$ the set of solutions. The differential $\partial_{(H, J)}$ on $C F\left(H ; o_{N}, T_{q}^{*} N\right)$ is provided by the moduli space of solutions of the perturbed Cauchy-Riemann equation

$$
\left\{\begin{array}{l}
\frac{\partial u}{\partial \tau}+J\left(\frac{\partial u}{\partial t}-X_{H}(u)\right)=0 \\
u(\tau, 0) \in o_{N}, u(\tau, 1) \in T_{q}^{*} N .
\end{array}\right.
$$

An element $\alpha \in C F\left(H ; o_{N}, T_{q}^{*} N\right)$ is expressed as a finite sum

$$
\alpha=\sum_{z \in \mathcal{C} h o r d\left(H ; o_{N}, T_{q}^{*} N\right)} a_{z}[z], \quad a_{z} \in \mathbb{Z} .
$$

We denote the level of the chain $\alpha$ by

$$
\lambda_{H}(\alpha):=\max _{z \in \operatorname{supp} \alpha}\left\{\mathcal{A}_{H}^{c l}(z)\right\} .
$$

The resulting invariant $\rho(H ;\{q\})$ is to be defined by the mini-max value

$$
f_{H}(q):=\inf _{\alpha \in[q]} \lambda_{H}(\alpha)
$$


where $[q] \in H_{0}(\{q\} ; \mathbb{Z})$ is a generator of the homology group $H_{0}(\{q\} ; \mathbb{Z})$.

Equivalently, we can consider the pair $\left(L_{H}, T_{q}^{*} N\right)$ for the action functional

$$
\mathcal{A}^{(0)}(\gamma):=\int \gamma^{*} \theta+h_{H}(\gamma(0))
$$

defined on $\Omega\left(L_{H}, T_{q}^{*} N\right)$ which defines the geometric version of the Floer complex $C F\left(L_{H}, T_{q}^{*} N\right)$ via the equation

$$
\left\{\begin{array}{l}
\frac{\partial v}{\partial \tau}+J_{0} \frac{\partial v}{\partial t}=0 \\
v(\tau, 0) \in L_{H}, v(\tau, 1) \in T_{q}^{*} N
\end{array}\right.
$$

Now by the same argument performed in sections 3 and 4 , we can localize the Floer complex to $C F\left(L_{H}, T_{q}^{*} N ; V\right)$ and define the local version of the spectral invariant $\rho_{V}^{\operatorname{lag}}(H ;\{q\})$ by

$$
f_{H}^{V}(q)=\inf _{\alpha \in[q]} \lambda_{H}(\alpha)
$$

where $[q] \in H_{0}(\{q\} ; \mathbb{Z})$ is a generator of the homology group $H_{0}(\{q\} ; \mathbb{Z})$. By varying $q \in N$, this defines a function $f_{H}^{V}: N \rightarrow \mathbb{R}$ which is precisely the local version of the basic phase function defined in [Oh3]. We denote the associated graph part of the front $W_{R_{H}}$ of the $L_{H}$ by $G_{f_{H}^{V}}$.

We summarize the main properties of $f_{H}^{V}$ whose proofs are verbatim the same as those established for the (global) basic function $f_{H}$ in [Oh3], [Oh14] by replacing the global Floer complex $C F_{*}(H)$ by the local complex $C F_{*}^{[i d]}(H ; V)$. First we have

Theorem 6.5. Let $H=H(t, x) \in \mathcal{H}_{\delta}^{\text {engulf }}\left(T^{*} N\right)$ and the Lagrangian submanifold $L_{H}=\phi_{H}^{1}\left(o_{N}\right)$. Consider the function $f_{H}^{V}$ defined above. Then for any $x \in L_{H}$

$$
f_{H}^{V}(\pi(x))=h_{H}(x)=\mathcal{A}_{H}^{c l}\left(z_{x}^{H}\right)
$$

for some Hamiltonian chord $z_{x}^{H}$ ending at $L_{H} \cap T_{\pi(x)}^{*} N$.

Once we have achieved localizations of various entities arising in Floer complex in the previous subsection, the following equality can be proven by the same argument used in the proof of Theorem 6.2 using the localized version of Lagrangian spectral invariants and basic phase function. We omit the details of its proof. 
Theorem 6.6. Let $V \subset T^{*} N$ be as before. Then

$$
f_{H}^{V}=f_{H}
$$

for any $V$-engulfed $H$.

An immediate corollary of this theorem is the following inequality.

Corollary 6.7. For any Hamiltonian $H \in \mathcal{H}_{\delta}^{\text {engulf }}\left(T^{*} N\right)$,

$$
\max f_{H}^{V} \leq E^{-}(H) .
$$

Furthermore if $H, H^{\prime} \in \mathcal{H}_{\delta}^{\text {engulf }}\left(T^{*} N\right)$

$$
\left\|f_{H}^{V}-f_{H^{\prime}}^{V}\right\|_{\infty} \leq\left\|H-H^{\prime}\right\| .
$$

\subsection{Localization of triangle product}

A version of localization of triangle product was previously exploited in [Se, Sp, Oh10] for smooth Hamiltonians.

Instead of delving into the localization of triangle product in full generality, we will restrict ourselves to the case of the zero section $o_{L}$ in the cotangent bundle. Once we isolate the invariant set into a Darboux neighborhood $U \subset M$, we may identify $U$ with a neighborhood $V$ of the zero section $o_{L} \subset T^{*} L$ and consider a Hamiltonian $F$ with $\operatorname{supp} F \subset V$. It then follows that due to the non-presence of bubbling effect for the pair $\left(T^{*} L, o_{L}\right)$, by an easier argument, we obtain the decomposition $\partial=\partial_{(0)}+\partial^{\prime}$ of the Floer differential $\partial$ on $C F_{*}\left(F ; T^{*} L\right)$, and obtain the local Floer complex

$$
\left(C F_{*}^{[i d]}\left(o_{L}, F ; V\right), \partial_{(0)}\right) .
$$

We first recall the definition of the triangle product described in [Oh4], [FO] and the discussion carried out in section 8 [Oh14]. Similar idea of localizing the triangle product was used in [Se], [Oh10] and [Sp]. Instead of delving into the localization in full generality, we restrict ourselves to the case relevant to our main interest arising from the study in [Oh14].

Let $q \in N$ be given. Consider the Hamiltonians $H:[0,1] \times T^{*} N \rightarrow \mathbb{R}$ such that $L_{H}$ intersects transversely both $o_{N}$ and $T_{q}^{*} N$. We consider the Floer complexes

$$
C F\left(L_{H}, o_{N}\right), \quad C F\left(o_{N}, T_{q}^{*} N\right), \quad C F\left(L_{H}, T_{q}^{*} N\right)
$$


each of which carries filtration induced from the effective action function given below. We denote by $\mathfrak{v}(\alpha)$ the level of the chain $\alpha$ in any of these complexes.

More precisely, $C F\left(L_{H}, o_{N}\right)$ is filtered by the effective functional

$$
\mathcal{A}^{(1)}(\gamma):=\int \gamma^{*} \theta+h_{H}(\gamma(0))
$$

$C F^{\mu}\left(o_{N}, T_{q}^{*} N\right)$ by

$$
\mathcal{A}^{(2)}(\gamma):=\int \gamma^{*} \theta
$$

and $C F\left(L_{H}, T_{q}^{*} N\right)$ by

$$
\mathcal{A}^{(0)}(\gamma):=\int \gamma^{*} \theta+h_{H}(\gamma(0))
$$

respectively. We recall the readers that $h_{H}$ is the potential of $L_{H}$ and the zero function the potentials of $o_{N}, T_{q}^{*} N$.

We now consider the triangle product in the chain level, which we denote by

$$
\mathfrak{m}_{2}: C F\left(L_{H}, o_{N}\right) \otimes C F\left(o_{N}, T_{q}^{*} N\right) \rightarrow C F\left(L_{H}, T_{q}^{*} N\right)
$$

following the general notation from [FOOO1]. This product is defined by considering all triples

$$
x_{1} \in L_{H} \cap o_{N}, x_{2} \in o_{N} \cap T_{q}^{*} N, x_{0} \in L_{H} \cap T_{q}^{*} N
$$

with the polygonal Maslov index $\mu\left(x_{1}, x_{2} ; x_{0}\right)$ whose associated analytical index, or the virtual dimension of the moduli space

$$
\mathcal{M}_{3}\left(D^{2} ; x_{1}, x_{2} ; x_{0}\right):=\widetilde{\mathcal{M}}_{3}\left(D^{2} ; x_{1}, x_{2} ; x_{0}\right) / \operatorname{PSL}(2, \mathbb{R})
$$

of $J$-holomorphic triangles, becomes zero and counting the number of elements thereof.

Definition 6.8. Let $J=J(z)$ be a domain-dependent family of compatible almost complex structures with $z \in D^{2}$. We define the space $\widetilde{\mathcal{M}}_{3}\left(D^{2} ; x_{1}, x_{2} ; x_{0}\right)$ by the pairs $\left(w,\left(z_{0}, z_{1}, z_{2}\right)\right)$ that satisfy the following:

(1) $w: D^{2} \rightarrow T^{*} N$ is a continuous map satisfying $\bar{\partial}_{J} w=0$ on $D^{2} \backslash$ $\left\{z_{0}, z_{1}, z_{2}\right\}$, 
(2) the marked points $\left\{z_{0}, z_{1}, z_{2}\right\} \subset \partial D^{2}$ with counter-clockwise cyclic order,

(3) $w\left(z_{1}\right)=x_{1}, w\left(z_{2}\right)=x_{2}$ and $w\left(z_{0}\right)=x_{0}$,

(4) the map $w$ satisfies the Lagrangian boundary condition

$$
w\left(\partial_{1} D^{2}\right) \subset L_{H}, w\left(\partial_{2} D^{2}\right) \subset o_{N}, w\left(\partial_{3} D^{2}\right) \subset T_{q}^{*} N
$$

where $\partial_{i} D^{2} \subset \partial D^{2}$ is the are segment in between $x_{i}$ and $x_{i+1}(i$ $\bmod 3)$.

We have the following energy estimate

Proposition 6.9 (Proposition 8.2 [Oh14]). Suppose $w: D^{2} \rightarrow T^{*} N$ be any smooth map with finite energy that satisfy all the conditions given in Definition 6.8, but not necessarily J-holomorphic. We denote by $c_{x}:[0,1] \rightarrow$ $T^{*} N$ the constant path with its value $x \in T^{*} N$. Then we have

$$
\int w^{*} \omega_{0}=\mathcal{A}^{(1)}\left(c_{x_{1}}\right)+\mathcal{A}^{(2)}\left(c_{x_{2}}\right)-\mathcal{A}^{(0)}\left(c_{x_{0}}\right)
$$

An immediate corollary of this proposition from the definition of $\mathfrak{m}_{2}$ is that the map (6.8) restricts to

$$
\mathfrak{m}_{2}: C F^{\lambda}\left(L_{H}, o_{N}\right) \otimes C F^{\mu}\left(o_{N}, T_{q}^{*} N\right) \rightarrow C F^{\lambda+\mu}\left(L_{H}, T_{q}^{*} N\right)
$$

and in turn induces the product map

$$
*_{F}: H F^{\lambda}\left(L_{H}, o_{N}\right) \otimes H F^{\mu}\left(o_{N}, T_{q}^{*} N\right) \rightarrow H F^{\lambda+\mu}\left(L_{H}, T_{q}^{*} N\right)
$$

in homology. This is because if $w$ is $J$-holomorphic $\int w^{*} \omega \geq 0$. This ends the summary of triangle product on the global Floer complex explained in [Oh14].

To localize the above construction to obtain the local analogs

$$
\mathfrak{m}_{2,(0)}: C F^{\lambda}\left(L_{H}, o_{N} ; V\right) \otimes C F^{\mu}\left(o_{N}, T_{q}^{*} N ; V\right) \rightarrow C F^{\lambda+\mu}\left(L_{H}, T_{q}^{*} N ; V\right)
$$

and the induced product

$$
{ }_{F,(0)}^{*}: H F^{\lambda}\left(L_{H}, o_{N} ; V\right) \otimes H F^{\mu}\left(o_{N}, T_{q}^{*} N ; V\right) \rightarrow H F^{\lambda+\mu}\left(L_{H}, T_{q}^{*} N ; V\right)
$$

in homology, we have only to prove the analog to Theorem 3.1 and Theorem 4.1 for the moduli space

$$
\widetilde{\mathcal{M}}_{3}\left(D^{2} ; x_{1}, x_{2} ; x_{0}\right)
$$


Theorem 6.10. Let $V$ be an open neighborhood of the zero section $o_{L}$ and let $H \in \mathcal{H}_{\delta}^{\text {engulf }}\left(T^{*} L\right)$. Then for any given open neighborhood $V$ of $o_{L}$, there exists some $\delta_{0}>0$ such that for any $0<\delta \leq \delta_{0}$, for any element $w \in \widetilde{\mathcal{M}}_{3}\left(L_{H}, o_{N}, T_{q}^{*} N\right)$ the following alternative holds:

(1) Image $w \subset V$ and $\max _{z \in \mathbb{R} \times\{0,1\}} d\left(v(z), o_{L}\right) \leq \delta$,

(2) Image $w \not \subset V$ and $\int w^{*} \omega \geq C\left(J_{0}, V\right)$.

Proof. The only difference in the proof of this theorem from Theorem 3.1 and 4.1 is that we also need to use the strong maximum principle along the fiber Lagrangian $T_{q}^{*} N$ in addition. We would like to note that the intersection

$$
T_{q}^{*} N \cap S^{\delta}\left(T^{*} N\right)
$$

is Legendrian and so a $J_{g}$-holomorphic curve satisfies strong maximum principle along $T_{q}^{*} N$. We refer to [EHS], [Oh3] for such an application of strong maximum principle to obtain $C^{0}$-estimate.

The proof is exactly the same as that of Theorem 3.1 and Theorem 4.1 and so omitted.

We define the 'thin' part of $\mathfrak{m}_{2}$ by counting those elements $w$ from $\widetilde{\mathcal{M}}_{3}\left(L_{H}, o_{N}, T_{q}^{*} N\right)$ of the type (1) above and decompose

$$
\mathfrak{m}_{2}=\mathfrak{m}_{2,(0)}+\mathfrak{m}_{2}^{\prime}
$$

It also follows that $\mathfrak{m}_{2,(0)}$ induces a product map

$$
\mathfrak{m}_{2,(0)}: C F^{\lambda}\left(L_{H}, o_{N} ; V\right) \otimes C F^{\mu}\left(o_{N}, T_{q}^{*} N ; V\right) \rightarrow C F^{\lambda+\mu}\left(L_{H}, T_{q}^{*} N ; V\right)
$$

It is straightforward to check that this map satisfies

$$
\partial_{(0)}\left(\mathfrak{m}_{2,(0)}(x, y)\right)=\mathfrak{m}_{2,(0)}\left(\partial_{(0)}(x), y\right) \pm \mathfrak{m}_{2,(0)}\left(x, \partial_{(0)}(y)\right)
$$

and so induces a product

$$
\left.*_{F,(0)}: H F_{*}^{\lambda}\left(L_{H}, o_{N} ; V\right) \otimes H F_{*}^{\mu}\left(o_{N}, T_{q}^{*} N ; V\right) \rightarrow H F_{*}^{\lambda+\mu}\left(L_{H}, T_{q}^{*} N ; V\right)\right)
$$

as in [Oh14]. 


\section{Appendix A. Local Floer complex of engulfed Hamiltonian $C^{0}$-approximate loop}

In this appendix, we give the construction of local Hamiltonian Floer complex in the context of $C^{0}$-small topological Hamiltonian loops for a future purpose. Exposition of this appendix closely follows that of section 4 [Oh5] except that we need to explain the points, if necessary, about why $C^{0}$ smallness of $\phi_{F}$ is enough to localize the Floer complex of the fixed point set of $\phi_{F}^{1}$.

\section{A.1. Hamiltonian Floer complex}

This section reviews the standard construction in Hamiltonian Floer theory. We closely follow exposition of chapter 2 [FOOO2] for some enhancement added which is useful for our purpose later.

Let $\widetilde{\mathcal{L}}_{0}(M)$ be the set of all the pairs $[\gamma, w]$ where $\gamma$ is a loop $\gamma: S^{1} \rightarrow M$ and $w: D^{2} \rightarrow M$ a disc with $\left.w\right|_{\partial D^{2}}=\gamma$. We identify $[\gamma, w]$ and $\left[\gamma^{\prime}, w^{\prime}\right]$ if $\gamma=\gamma^{\prime}$ and $w$ is homotopic to $w^{\prime}$ relative to the boundary $\gamma$. When a one-periodic Hamiltonian $H:(\mathbb{R} / \mathbb{Z}) \times M \rightarrow \mathbb{R}$ is given, we consider the perturbed functional $\mathcal{A}_{H}: \widetilde{\mathcal{L}}_{0}(M) \rightarrow \mathbb{R}$ defined by

$$
\mathcal{A}_{H}([\gamma, w])=-\int w^{*} \omega-\int H(t, \gamma(t)) d t
$$

For a Hamiltonian $H:[0,1] \times M \rightarrow \mathbb{R}$, we denote its flow, a Hamiltonian isotopy, by $\phi_{H}: t \mapsto \phi_{H}^{t} \in \operatorname{Ham}(M, \omega)$. We denote the time-one map by $\phi_{H}^{1}$. We put

$$
\operatorname{Fix} \phi_{H}^{1}=\left\{p \in M \mid \phi_{H}^{1}(p)=p\right\} .
$$

Each element $p \in \operatorname{Per}(H)$, the set of 1-periodic orbits, induces a map $z_{x}=z_{x}^{H}: S^{1} \rightarrow M$, by the correspondence

$$
z_{x}^{H}(t)=\phi_{H}^{t}\left(\left(\phi_{H}^{1}\right)^{-1}(x)\right),
$$

where $t \in \mathbb{R} / \mathbb{Z} \cong S^{1}$.

We denote by $\operatorname{Per}(H)$ the set of one-periodic solutions of $\dot{x}=X_{H}(t, x)$. Then (A.2) provides a one-one correspondence between $\operatorname{Fix} \phi_{H}^{1}$ and $\operatorname{Per}(H)$. The set of critical points of $\mathcal{A}_{H}$ is given by

$$
\operatorname{Crit}\left(\mathcal{A}_{H}\right)=\left\{[z, w]|\gamma \in \operatorname{Per}(H), w|_{\partial D^{2}}=\gamma\right\} .
$$


We consider the universal (downward) Novikov field

$$
\Lambda=\left\{\sum_{i=1}^{\infty} a_{i} T^{\lambda_{i}} \mid a_{i} \in \mathbb{R}, \lambda_{i} \rightarrow-\infty\right\}
$$

and define a valuation $\mathfrak{v}_{T}$ on $\Lambda$ by

$$
\mathfrak{v}_{T}\left(\sum_{i=1}^{\infty} a_{i} T^{\lambda_{i}}\right)=\sup \left\{\lambda_{i} \mid a_{i} \neq 0\right\} .
$$

It satisfies the following properties:

(1) $\mathfrak{v}_{T}(x y)=\mathfrak{v}_{T}(x)+\mathfrak{v}_{T}(y)$,

(2) $\mathfrak{v}_{T}(x+y) \leq \max \left\{\mathfrak{v}_{T}(x), \mathfrak{v}_{T}(y)\right\}$,

(3) $\mathfrak{v}_{T}(x)=-\infty$ if and only if $x=0$,

(4) $\mathfrak{v}_{T}(q)=1$

(5) $\mathfrak{v}_{T}(a x)=\mathfrak{v}_{T}(x)$ if $a \in R \backslash\{0\}$.

We consider the $\Lambda$ vector space $\widehat{C F}(H ; \Lambda)$ with basis given by the critical point set $\operatorname{Crit}\left(\mathcal{A}_{H}\right)$ of $\mathcal{A}_{H}$.

Definition A.1. We define an equivalence relation $\sim$ on $\widehat{C F}(H ; \Lambda)$ so that $[z, w] \sim T^{c}\left[z^{\prime}, w^{\prime}\right]$ if and only if

$$
z=z^{\prime}, \int_{D^{2}} w^{\prime *} \omega=\int_{D^{2}} w^{*} \omega-c .
$$

The quotient of $\widehat{C F}(H ; \Lambda)$ modded out by this equivalence relation $\sim$ is called the Floer complex of the periodic Hamiltonian $H$ and denoted by $C F(H ; \Lambda)$.

Here we do not assume the condition on the Conley-Zehnder indices and work with $\mathbb{Z}_{2}$-grading. In the standard literature on Hamiltonian Floer homology, an additional requirement

$$
c_{1}\left(\bar{w} \# w^{\prime}\right)=0
$$

is commonly imposed in the definition Floer complex, denoted by $C F(H)$. For the purpose of the current paper similarly as in [FOOO2], the equivalence relation (A.4) is enough and more favorable in that it makes the associated Novikov ring becomes a field. To differentiate the current definition from $C F_{*}(H)$, we denote the complex used in the present paper by $C F_{*}(H ; \Lambda)$. 
Lemma A.2. As a $\Lambda$ vector space, $C F_{*}(H ; \Lambda)$ is isomorphic to the direct sum $\Lambda^{\# \operatorname{Per}(H)}$.

Moreover the following holds: We fix a lifting $\left[z, w_{z}\right] \in \operatorname{Crit}\left(\mathcal{A}_{H}\right)$ for each $z \in \operatorname{Per}(H)$. Then any element $x$ of $C F(M, H ; \Lambda)$ is uniquely written as a sum

$$
x=\sum_{z \in \operatorname{Per}(H)} x_{z}\left[z, w_{z}\right], \quad \text { with } x_{z} \in \Lambda .
$$

Definition A.3. (1) Let $x$ be as in (A.5). We define

$$
\mathfrak{v}_{T}(x)=\max \left\{\mathfrak{v}_{T}\left(x_{z}\right)+\mathcal{A}_{H}\left(\left[z, w_{z}\right]\right) \mid \gamma \in \operatorname{Per}(H)\right\} .
$$

(2) We define a filtration $F^{\lambda} C F(M, H ; \Lambda)$ on $C F(M, H ; \Lambda)$ by

$$
F^{\lambda} C F(H ; \Lambda)=\left\{x \in C F(H ; \Lambda) \mid \mathfrak{v}_{T}(x) \leq \lambda\right\} .
$$

We have

$$
F^{\lambda_{1}} C F(H ; \Lambda) \subset F^{\lambda_{2}} C F(H ; \Lambda)
$$

if $\lambda_{1}<\lambda_{2}$. We also have

$$
\bigcap_{\lambda} F^{\lambda} C F(H ; \Lambda)=\{0\}, \quad \bigcup_{\lambda} F^{\lambda} C F(H ; \Lambda)=C F(M ; H) .
$$

(3) We define a metric $d_{T}$ on $C F(H ; \Lambda)$ by

$$
d_{T}\left(x, x^{\prime}\right)=e^{\mathfrak{v}_{T}\left(x-x^{\prime}\right)} .
$$

Then (A.3), (A.4) and Definition A.3 imply that

$$
\mathfrak{v}_{T}(a \mathfrak{x})=\mathfrak{v}_{T}(a)+\mathfrak{v}_{T}(\mathfrak{x})
$$

for $a \in \Lambda^{\downarrow}, \mathfrak{x} \in C F(H ; \Lambda)$. We also have

$$
T^{\lambda_{1}} \cdot F^{\lambda_{2}} C F(H ; \Lambda) \subseteq F^{\lambda_{1}+\lambda_{2}} C F(H ; \Lambda) .
$$

Lemma A.4. (1) $\mathfrak{v}_{T}$ is independent of the choice of the lifting $z \mapsto\left[z, w_{z}\right]$.

(2) $C F\left(H ; \Lambda^{\downarrow}\right)$ is complete with respect to the metric $d_{T}$.

(3) The infinite sum

$$
\sum_{[z, w] \in \operatorname{Crit} \mathcal{A}_{H}} x_{[z, w]}[z, w]
$$


converges in $C F\left(H ; \Lambda^{\downarrow}\right)$ with respect to the metric $d_{T}$ if

$$
\left\{[z, w] \in \operatorname{Crit} \mathcal{A}_{H} \mid \mathfrak{v}_{T}\left(x_{[z, w]}\right)+\mathcal{A}_{H}([z, w])>-C, x_{[z, w]} \neq 0\right\} .
$$

is finite for any $C \in \mathbb{R}$.

\section{A.2. Isolating local Hamiltonian Floer complex}

This section is a modification of section 4.1 [Oh5] which treats the case of $C^{2}$-small perturbation of Hamiltonians $H$ following section 3 [Oh2].

As in section 2, we will replace the condition of $\phi_{F}$ being $C^{1}$-small by $\phi_{F}$ being $C^{0}$-small with the same kind of bound on the Hofer norm $\|F\|$. Once we have established the thick-thin dichotomy given in Theorem 3.1, we can safely repeat the arguments laid out in section 4.1 [Oh5], whose summary is now in order.

For given such $F$, we consider the subset $\mathcal{U}=\mathcal{U}\left(U_{\Delta}\right) \subset \mathcal{L}_{0}(M)$ of loops given by

$$
\mathcal{U}=\left\{\gamma \in \mathcal{L}_{0}(M) \mid(\gamma(t), \gamma(0)) \in U_{\Delta}\right\} .
$$

for a fixed Darboux neighborhood $U_{\Delta}$ of the diagonal $\Delta \subset M \times M$ for all $t \in[0,1]$. In particular, any periodic orbit $z$ of the flow $\phi_{H}$ is contained in $\mathcal{U} \subset \mathcal{L}(M)$ has a canonical isotopy class of contraction $w_{z}$. We will always use this convention $w_{z}$ whenever there is a canonical contraction of $z$ like in this case of small loops. This provides a canonical embedding of $\mathcal{U} \subset \widetilde{\mathcal{L}_{0}}(M)$ defined by

$$
z \rightarrow\left[z, w_{z}\right] .
$$

We denote this canonical embedding by $\mathcal{U}^{[i d]}$. This selects a distinguished component of

$$
\pi^{-1}(\mathcal{U}) \subset \widetilde{\mathcal{L}}_{0}(M)
$$

and other components can be given by

$$
\mathcal{U}^{[g]}=g \cdot \mathcal{U}^{[i d]}, \quad g \in \Gamma_{\omega}
$$

similarly as before.

Combining the constructions from [Oh5] and section 2, we give

Definition A.5. Let $J=\left\{J_{t}\right\}$ with $\left|J_{t}-J_{0}\right|_{C^{1}}<\varepsilon_{3}$ with $\varepsilon_{3}$ sufficiently small. For any $F \in \mathcal{H}_{\delta}^{\text {engulf }}(M)$ and for the given Darboux neighborhood $U_{\Delta}$ of the diagonal $\Delta \subset M \times M$ such that

$$
\phi_{F}^{t}(\Delta) \subset \operatorname{Int} U_{\Delta},
$$


we define

$$
\mathcal{M}^{[g]}(F, J ; \mathcal{U})=\left\{u \in \mathcal{M}(F, J) \mid(u(\tau)(t), u(\tau)(0)) \in \operatorname{Int} U_{\Delta}^{[g]} \text { for all } \tau\right\}
$$

for each $g \in \Gamma_{\omega}$. Consider the evaluation map

$$
e v: \mathcal{M}\left(F, J: \mathcal{U}^{[g]}\right) \rightarrow \mathcal{U} \subset \mathcal{L}_{0}(M) ; \quad e v(u)=u(0) .
$$

For each open neighborhood $U_{\Delta} \subset M \times M$ of $\Delta \subset U_{\Delta}$, we define the local Floer complex in $\mathcal{U}^{[g]}$ by

$$
\mathcal{S}\left(F, J ; \mathcal{U}^{[g]}\right):=e v\left(\mathcal{M}\left(F, J ; \mathcal{U}^{[g]}\right) \subset \mathcal{L}_{0}(M) .\right.
$$

We say $\mathcal{S}\left(F, J ; \mathcal{U}^{[g]}\right)$ is isolated in $\mathcal{U}^{[g]}$ if its closure is contained in $\mathcal{U}^{[g]}$.

Using Theorem 5.2, we define the local Floer homology, denoted by $H F^{[g]}(F, J ; \mathcal{U})$. Furthermore, the pull-back of the action functional $\mathcal{A}_{F}$ to $\mathcal{U}^{[g]}$ via the above mentioned embedding into $\widetilde{\mathcal{L}}_{0}(M)$ provides a filtration on the local Floer complex $C F^{[g]}(F ; \mathcal{U})$.

Therefore by considering the parameterized family

$$
\mathcal{S}\left(G^{s}, J ; \mathcal{U}^{[i d]}\right),
$$

the proof of Theorem 5.2 implies that if $G \in \mathcal{H}_{\delta}^{\text {engulf }}(M)$ and $\delta$ sufficiently small, $\mathcal{S}\left(J, G^{s}: \mathcal{U}^{[i d]}\right)$ are isolated in $\mathcal{U}^{[i d]}$ for all $s$ and its homology is isomorphic to $H_{*}(M ; R)$. For readers' convenience, we provide the detailed comparison argument between the Hamiltonian Floer complex of Fix $\phi_{G}^{1}$ and the Lagrangian Floer complex of the pair $\left(\Delta\right.$, Graph $\left.\phi_{G}^{1}\right)$ in Appendix borrowing from that of section $4.2[\mathrm{Oh} 5]$.

\section{A.3. Fix $\phi_{G}^{1}$ versus $\Delta \cap \operatorname{graph} \phi_{G}^{1}$}

The main goal of this sub-section is to compare the Hamiltonian Floer homology of $G$ with the Lagrangian Floer complex between $\Delta$ and graph $\phi_{G}^{1}$ in the product $(M, \omega) \times(M,-\omega)$ when $G \in \mathcal{H}_{\delta}^{\text {engulf }}(M)$ with $\delta$ sufficiently small.

We now compare the local Floer homology $H F^{[i d]}(J, G: \mathcal{U})$ of $G \in$ $\mathcal{H}_{\delta}^{\text {engulf }}(M)$ and two versions of its intersection counterparts, one $H F_{J_{0} \oplus-J_{0}, 0}^{[i d]]}\left(\operatorname{Graph} \phi_{G}^{1}, \Delta: U_{\Delta}\right)$ and the other $H F_{\left(\phi_{G}\right)^{*} J_{0} \oplus-J_{0}, 0 \oplus G}^{[i] d}(\Delta, \Delta$ : $\left.U_{\Delta}\right)$. 
First we note that the two Floer complexes $\mathcal{M}_{J_{0} \oplus-J_{0}, 0}\left(\operatorname{Graph} \phi_{G}^{1}, \Delta\right.$ : $\left.\mathcal{U}^{[i d]}\right)$ and $\mathcal{M}_{\left(\phi_{G}\right)^{*} J_{0} \oplus-J_{0}, 0 \oplus G}\left(\Delta, \Delta: \mathcal{U}^{[i d]}\right)$ are canonically isomorphic by the assignment

$$
(\gamma(t), \gamma(t)) \mapsto\left(\left(\phi_{G}^{t}\right)^{-1}(\gamma)(t), \gamma(t)\right) .
$$

and so the two Lagrangian intersection Floer homology are canonically isomorphic: Here the above two moduli spaces are the solutions sets of the following Cauchy-Riemann equations

$$
\left\{\begin{array}{l}
\frac{\partial U}{\partial \tau}+\left(J_{0} \oplus-J_{0}\right) \frac{\partial U}{\partial t}=0 \\
U(\tau, 0) \in \operatorname{graph} \phi_{G}^{1}, U(\tau, 1) \in \Delta
\end{array}\right.
$$

and

$$
\left\{\begin{array}{l}
\frac{\partial U}{\partial \tau}+\left(\left(\phi_{G}^{1}\right)^{*} J_{0}\right) \oplus\left(-J_{0}\right)\left(\frac{\partial U}{\partial t}-X_{0 \oplus G}(U)\right)=0 \\
U(\tau, 0) \in \Delta, U(\tau, 1) \in \Delta
\end{array}\right.
$$

respectively, where $U=\left(u_{1}, u_{2}\right): \mathbb{R} \times[0,1] \rightarrow M \times M$. The relevant action functionals for these cases are given by

$$
\mathcal{A}_{0}([\Gamma, W])=-\int W^{*}(\omega \oplus-\omega)
$$

on $\widetilde{\Omega}\left(\operatorname{Graph} \phi_{G}^{1}, \Delta: M \times M\right)$ and

$$
\mathcal{A}_{0 \oplus G}([\Gamma, W])=\mathcal{A}_{0}(\Gamma, W)-\int_{0}^{1}(0 \oplus G)(\Gamma(t), t) d t
$$

on $\widetilde{\Omega}(\Delta, \Delta: M \times M)$ where we denote

$\Omega\left(\operatorname{Graph} \phi_{G}^{1}, \Delta: M \times M\right)=\left\{\Gamma:[0,1] \rightarrow M \times M \mid \Gamma(0) \in \operatorname{graph} \phi_{G}^{1}, \Gamma(1) \in \Delta,\right\}$

and similarly for $\Omega\left(\operatorname{Graph} \phi_{G}^{1}, \Delta: M \times M\right)$. Again the 'tilde' means the covering space which can be represented by the set of pairs $[\Gamma, W]$ in a similar way. The relations between the action functionals (A.7), (A.8) and $\mathcal{A}_{G}$ are evident and respect the filtration under the natural correspondences.

Next we will attempt to compare

$$
H F^{[i d]}(G, J ; \mathcal{U}), \quad H F_{J_{0} \oplus-J_{0}, G \oplus 0}^{[i d]}\left(\Delta, \Delta: U_{\Delta}\right) .
$$

Without loss of any generality, we will concern Hamiltonians $G$ such that $G \equiv 0$ near $t=0$, 1 , which one can always achieve by perturbing $G$ without changing its time-one map. 
There is no direct way of identifying the corresponding Floer complexes between the two.

As an intermediate case, we consider the Hamiltonian $G^{\prime}: M \times[0,1]$ defined by

$$
G^{\prime}(x, t)= \begin{cases}2 G(x, 2 t) & \text { for } 0 \leq t \leq \frac{1}{2} \\ 0 & \text { for } \frac{1}{2} \leq t \leq 1\end{cases}
$$

and the assignment

$$
\left(u_{0}, u_{1}\right) \in \mathcal{M}_{J_{0} \oplus-J_{0}, G \oplus 0}^{[i d]}\left(\Delta, \Delta: U_{\Delta}\right) \mapsto v \in \mathcal{M}\left(J, G^{\prime}: \mathcal{U}^{[i d]}\right)
$$

with $v(\tau, t):=u_{0} \# \bar{u}_{1}(\tau, t)$. Here the map $u_{0} \# \bar{u}_{1}:[0,1] \rightarrow M$ is the map defined by

$$
u_{0} \# \bar{u}_{1}(\tau, t)= \begin{cases}u_{0}(2 \tau, 2 t) & \text { for } 0 \leq t \leq \frac{1}{2} \\ u_{1}(2 \tau, 1-2 t) & \text { for } \frac{1}{2} \leq t \leq 1\end{cases}
$$

is well-defined and continuous because

$$
\begin{aligned}
& u_{0}(\tau, 1)=u_{0}(\tau, 0)=\bar{u}_{1}(\tau, 0) \\
& \bar{u}_{1}(\tau, 1)=u_{0}(\tau, 1)=u_{0}(\tau, 0) .
\end{aligned}
$$

Furthermore near $t=0,1$, this is smooth (and so holomorphic) by the elliptic regularity since $G^{\prime}$ is smooth (Recall that we assume that $G \equiv 0$ near $t=0,1$. Conversely, any element $v \in \mathcal{M}\left(J, G^{\prime}: \mathcal{U}^{[i d]}\right)$ can be written as the form of $u_{0} \# \bar{u}_{1}$ which is uniquely determined by $v$. This proves that (A.9) is a diffeomorphism from $\mathcal{M}_{J_{0} \oplus-J_{0}, G \oplus 0}^{[i d]}\left(\Delta, \Delta: U_{\Delta}\right)$ to $\mathcal{M}\left(J, G^{\prime}: \mathcal{U}^{[i d]}\right)$ which induces a filtration-preserving isomorphism between $H F_{J_{0} \oplus-J_{0}, G \oplus 0}^{[i d]}(\Delta, \Delta$ : $\left.U_{\Delta}\right)$ and $H F\left(J, G^{\prime}: \mathcal{U}^{[i d]}\right)$.

Finally, we need to relate $H F\left(J, G: \mathcal{U}^{[i d]}\right)$ and $H F\left(J, G^{\prime}: \mathcal{U}^{[i d]}\right)$. For this we note that $G$ and $G^{\prime}$ can be connected by a one-parameter family $G^{\text {para }}=\left\{G^{s}\right\}_{0 \leq s \leq 1}$ with

$$
G^{s}(x, t):= \begin{cases}\frac{2}{1+s} G\left(x, \frac{2}{1+s} t\right) & \text { for } 0 \leq t \leq \frac{s}{2} \\ 0 & \text { for } \frac{s}{2} \leq t \leq 1 .\end{cases}
$$

And we have

$$
\phi_{G^{s}}^{1}=\phi_{G}^{1} \quad \text { for all } s \in[0,1] .
$$


Therefore their spectra coincide, i.e., $\operatorname{Spec}(G)=\operatorname{Spec}\left(G^{\prime s}\right)=\operatorname{Spec}\left(G^{\prime}\right)$. Then there exists an isomorphism

$$
h_{G^{\text {para }}, J}^{a d b}: C F\left(G^{\prime}: \mathcal{U}^{[i d]}\right) \rightarrow C F\left(G: \mathcal{U}^{[i d]}\right)
$$

respects the filtration and so the induced homomorphism in its homology

$$
h_{G^{\text {para }}, J}^{a d b}: H F\left(J, G^{\prime}: \mathcal{U}^{[i d]}\right) \rightarrow H F\left(J, G: \mathcal{U}^{[i d]}\right)
$$

becomes a filtration-preserving isomorphism. See [K], [U], [Oh11] for such a construction.

\section{References}

[Che1] Y. Chekanvo, Hofer's symplectic energy and Lagrangian intersections, in Contact and Symplectic Geometry (Cambridge, 1994), ed. C.B. Thomas, Publ. Newtwon Insti. 8 Cambridge University Press, Cambridge, 1996, 296-306.

[Che2] Y. Chekanov, Lagrangian intersections, symplectic energy, and areas of holomorphic curves, Duke Math. J. 95 (1998), 213-226. MR1646550

[Co] C. Conley, Isolated Invariant Sets and the Morse Index, CBMS Regional Conference Series in Mathematics 38, American Mathematical Society, Providence, R.I., 1978. MR0511133

[EHS] Y. Eliashberg, H. Hofer, and D. Salamon, Lagrangian intersections in contact geometry, Geom. Funct. Anal. 5 (1995), 244-269. MR1334868

[F11] A. Floer, Morse theory for Lagrangian intersections, J. Differential Geom. 28 (1988), no. 3, 513-547. MR0965228

[F12] A. Floer, Symplectic fixed points and holomorphic spheres, Commun. Math. Phys. 120 (1989), 575-611. MR0987770

[Fl3] A. Floer, Witten's complex and infinite-dimensional Morse theory, J. Differential Geom. 30 (1989), no. 1, 207-221. MR1001276

[FO] K. Fukaya, Y.-G. Oh, Zero-loop open strings in the cotangent bundle and Morse homotopy, Asian J. Math. 1 (1997), no. 1, 96-180. MR1480992 
[FOOO1] K. Fukaya, Y.-G. Oh, H. Ohta, K. Ono, Lagrangain Intersection Floer Theory; Anomaly and Obstruction, vol I \& II, AMS/IP Advanced Math Series, Providence, 2009. MR2548482

[FOOO2] K. Fukaya, Y.-G. Oh, H. Ohta, K. Ono, Spectral invariants with bulk, quasimorphisms and Lagrangian intersection Floer theory, preprint, arXiv:1105.5123. MR3076063

[FOOO3] K. Fukaya, Y.-G. Oh, H. Ohta, K. Ono, Displacement of polydisks and Lagrangian Floer theory, J. Symplectic Geom. 11 (2013), no. 2, 231-268, arXiv:1104.4267. MR3046491

[K] E. Kerman, Displacement energy of coisotropic submanifolds and Hofer's geometry, J. Mod. Dyn. 2 (2008), 471-497. MR2417482

[Oh1] Y.-G. Oh, Relative Floer and quantum cohomology and the symplectic topology of Lagrangian submanifolds, Proceedings for the 1994 Symplectic Topology program, - Contact and Symplectic Geometry - Publ. of the Newton Institute, eds. by C. B. Thomas, Cambridge University Press, Cambridge, England, 1996, pp. 201267. MR1432465

[Oh2] Y.-G. Oh, Floer cohomology, spectral sequences, and the Maslov class of Lagrangian embeddings, Internat. Math. Res. Notices (1996), no. 7, 305-346. MR1389956

[Oh3] Y.-G. Oh, Symplectic topology as the geometry of action functional, I, J. Differ. Geom. 46 (1997), 499-577. MR1484890

[Oh4] Y.-G. Oh, Symplectic topology as the geometry of action functional, II, Commun. Anal. Geom. 7 (1999), 1-55. MR1674121

[Oh5] Y.-G. Oh, Chain level Floer theory and Hofer's geometry of the Hamiltonian diffeomorphism group, Asian J. Math. 6 (2002), 579624; Erratum 7 (2003), 447-448. MR1958084

[Oh6] Y.-G. Oh, Construction of spectral invariants of Hamiltonian paths on closed symplectic manifolds, in "The Breadth of Symplectic and Poisson Geometry", Prog. Math. 232, 525-570, Birkhäuser, Boston, 2005. MR2103018

[Oh7] Y.-G. Oh, Spectral invariants, analysis of the Floer moduli space and geometry of Hamiltonian diffeomorphisms, Duke Math. J. 130 (2005), 199-295. MR2181090

[Oh8] Y.-G. Oh, Floer mini-max theory, the Cerf diagram and spectral invariants, J. Korean Math. Soc. 46 (2009), 363-447. MR2494501 
[Oh9] Y.-G. Oh, The group of Hamiltonian homeomorphisms and continuous Hamiltonian flows, Contemp. Math., 512, Amer. Math. Soc., Providence, RI, 2010, pp. 149-177. MR2605316

[Oh10] Y.-G. Oh, Seidel's long exact sequence on Calabi-Yau manifolds, Kyoto J. Math. 51 (2011), 687-765. MR2824005

[Oh11] Y.-G. Oh, Symplectic Topology and Floer Homology, book in preparation, available at http://math.wisc.edu/ oh/all.pdf.

[Oh12] Y.-G. Oh, Extension of Calabi homomorphism and nonsimpleness of the area-preserving homeomorphism group of $D^{2}$, preprint 2011 , arXiv.1010.1081 (withdrawn).

[Oh13] Y.-G. Oh, Homotopy invariance of spectral invariants of topological hamiltonian flows and its Lagrangian analog, preprint, arXiv:1111.5992 (withdrawn).

[Oh14] Y.-G. Oh, Geometry of generating functions and Lagrangian spectral invariants, submitted, arXiv:1206.4788.

[OM] Y.-G. Oh, S. Müller, The group of Hamiltonian homeomorphisms and $C^{0}$ symplectic topology, J. Symplectic Geom. 5 (2007), 167219. MR2377251

[Po] L. Polterovich, The Geometry of the Group of Symplectic Diffeomorphisms, Lectures in Math. ETH Zürich, Birkhäuser, Basel, 2001. MR1826128

[Se] P. Seidel, A long exact sequence for symplectic Floer cohomology, Topology 42 (2003), 1003-1063. MR1978046

[Sp] P. Spaeth, Length minimizing paths in the Hamiltonian diffeomorphism group, J. Symplectic Geom. 6 (2008), no. 2, 159-187. MR2434439

[U] M. Usher, Boundary depth in Floer theory and its applications to Hamiltonian dynamics and coisotropic submanifolds, Israel J. Math. 184 (2011), 1-57. MR2823968

YONG-GEun OH

IBS Center for Geometry and Physics

Institute For BasiC SCIENCES

POHANG

KOREA 
Department of Mathematics

POSTECH, POHANG

KOREA

Department of Mathematics

UNIVERSITY OF WINSCONSIN

MADISON, WI 53706

USA

E-mail address: yongoh@ibs.re.kr; oh@math.wisc.edu

Received September 10, 2013 